\title{
Global (weak) solution of the chemotaxis-Navier-Stokes equations with non-homogeneous boundary conditions and logistic growth
}

\author{
Marcel Braukhoff* \\ University of Paderborn, Warburgerstraße 100, 33098 Paderborn, Germany \\ Received 5 August 2015; received in revised form 25 February 2016; accepted 5 August 2016 \\ Available online 9 September 2016
}

\section{Abstract}

In biology, the behaviour of a bacterial suspension in an incompressible fluid drop is modelled by the chemotaxis-Navier-Stokes equations. This paper introduces an exchange of oxygen between the drop and its environment and an additionally logistic growth of the bacteria population. A prototype system is given by

$$
\left\{\begin{aligned}
n_{t}+u \cdot \nabla n & =\Delta n-\nabla \cdot(n \nabla c)+n-n^{2}, & & x \in \Omega, t>0, \\
c_{t}+u \cdot \nabla c & =\Delta c-n c, & & x \in \Omega, t>0, \\
u_{t} & =\Delta u+u \cdot \nabla u+\nabla P-n \nabla \varphi, & & x \in \Omega, t>0, \\
\nabla \cdot u & =0, & & x \in \Omega, t>0
\end{aligned}\right.
$$

in conjunction with the initial data $(n, c, u)(\cdot, 0)=\left(n_{0}, c_{0}, u_{0}\right)$ and the boundary conditions

$$
\frac{\partial c}{\partial v}=1-c, \frac{\partial n}{\partial v}=n \frac{\partial c}{\partial v}, u=0, \quad x \in \partial \Omega, t>0 .
$$

Here, the fluid drop is described by $\Omega \subset \mathbb{R}^{N}$ being a bounded convex domain with smooth boundary. Moreover, $\varphi$ is a given smooth gravitational potential.

Requiring sufficiently smooth initial data, the existence of a unique global classical solution for $N=2$ is proved, where $\|n\|_{L}{ }^{p}(\Omega)$ is bounded in time for all $p<\infty$, as well as the existence of a global weak solution for $N=3$.

(c) 2016 L'Association Publications de l'Institut Henri Poincaré. Published by Elsevier B.V. All rights reserved.

\section{Résumé}

Le comportement d'une suspension bactérienne dans une goutte de liquide incompressible est décrit par les équations de chemotaxis-Navier-Stokes. Cet article introduit un échange d'oxygène entre la goutte et son environnement et une croissance logistique de la population bactérienne. Le système généralise le prototype

\footnotetext{
* Present address: University of Cologne, Albertus-Magnus-Platz, 50923 Köln, Germany.

E-mail address: braukhoff@posteo.de.
} 


$$
\left\{\begin{aligned}
n_{t}+u \cdot \nabla n & =\Delta n-\nabla \cdot(n \nabla c)+n-n^{2}, & & x \in \Omega, t>0, \\
c_{t}+u \cdot \nabla c & =\Delta c-n c, & & x \in \Omega, t>0, \\
u_{t} & =\Delta u+u \cdot \nabla u+\nabla P-n \nabla \varphi, & & x \in \Omega, t>0, \\
\nabla \cdot u & =0, & & x \in \Omega, t>0
\end{aligned}\right.
$$

associé à la donnée initiale $(n, c, u)(\cdot, 0)=\left(n_{0}, c_{0}, u_{0}\right)$ et aux conditions du bord

$$
\frac{\partial c}{\partial v}=1-c, \frac{\partial n}{\partial v}=n \frac{\partial c}{\partial v}, u=0, \quad x \in \partial \Omega, t>0
$$

d'où $\Omega \subset \mathbb{R}^{N}$ soit un domaine borné et convexe avec un bord lisse. En outre, $\varphi$ soit un potentiel lisse gravitationnel. En supposant que la donnée initiale soit suffisamment régulière, on démontre l'existence d'une solution classique unique pour $N=2$ telle que $\|n\|_{L^{p}(\Omega)}$ est borné pour $p<\infty$ et l'existence d'une solution faible globale pour $N=3$.

(C) 2016 L'Association Publications de l'Institut Henri Poincaré. Published by Elsevier B.V. All rights reserved.

Keywords: Chemotaxis; Navier-Stokes; Non-homogeneous boundary conditions; Logistic growth

\section{Introduction}

In biology application concerning aerobic bacteria, the favour of the bacteria cells to a higher concentration of a chemical has been observed. This effect, called chemotaxis, is presumed to have an deep impact on the time evolution of a bacteria population. Depending on the kind of bacteria and the chemical, there are different concepts of chemotaxis whether the bacteria consumes or absorbs the chemical. In the present article, we focus on the model describing an oxygen-driven bacteria suspension swimming in an incompressible fluid like water which was firstly introduced by Tuval et al. [28].

The typical system mainly consists of three coupled subproblems: An equation for the population density $n$ of the bacteria, another for the concentration of oxygen $c$ and the Navier-Stokes equations describing the water flow $u$. It can be written as

$$
\left\{\begin{aligned}
n_{t}+u \cdot \nabla n & =\Delta n-\nabla \cdot(n \chi(c) \nabla c), & & x \in \Omega, t>0, \\
c_{t}+u \cdot \nabla c & =\Delta c-n f(c), & & x \in \Omega, t>0, \\
u_{t} & =v \Delta u+(u \cdot \nabla u)+\nabla P-n \nabla \varphi, & & x \in \Omega, t>0, \\
\nabla \cdot u & =0, & & x \in \Omega, t>0,
\end{aligned}\right.
$$

where $\Omega$ usually denotes the whole $\mathbb{R}^{N}$ or a bounded domain in $\mathbb{R}^{N}$ with a smooth boundary. The scalar valued parameter functions $\chi, f, \varphi$ and the constant $v>0$ are fixed. Here, a bounded domain is used to model a water drop. It has been carried out that the presence of a solvent for a chemotaxis system has a main influence on the migration of the population of the bacteria. In the case of $\Omega$ being a bounded domain, the system (1) requires boundary conditions. Tuval et al. propose Dirichlet boundary conditions for $u$, no-flux boundary conditions for $n$ and an constant rate of the oxygen dissolving into the drop. The mathematical literature $[10,21,26]$ adapts on the one hand the no flux boundary condition for $n$ from Tuval et al. [28]. On the other hand it assumes Neumann boundary conditions for $c$ neglecting the influence of the surroundings. However, Tuval et al. [28] emphasise the importance of the dissolving oxygen from the surroundings: According to the authors, it is an explanation for the observed migration of the bacteria population to the contact line between the drop and the air. Regarding this, the present article introduces nontrivial the boundary conditions for $c$, which are generalised to model a bidirectorial oxygen exchange between the water drop and the surroundings. Furthermore, it is the first mathematical article treating non-homogeneous boundary conditions for the oxygen equation in a chemotaxis system motivated by the experiment of Tuval et al.

Until now, there are several results for the solvability of (1) combined with the typical boundary conditions. We will recall some of the most important results concerning this setting of the chemotaxis-Navier-Stokes equations, which require $n_{0}, c_{0}$ being positive:

Assuming $\chi, f, \varphi$ are smooth functions with $f(0)=0$ and $f^{\prime} \geq 0$ on $\mathbb{R}$ and some additional regularity on $\varphi$, Duan et al. [9] prove a long-term small-data solution: Let $n_{\infty}$ be a constant; whenever $n_{0}-n_{\infty}, c_{0}, u_{0}$ are small enough (in 
$W^{3,2}\left(\mathbb{R}^{N}\right)$ ), then the system (1) admits a global unique classical solution on $\mathbb{R}^{N}$ for $N=2,3$. The authors also derive a time-decay of $\left(n-n_{\infty}, c, u\right)$ by using some assumptions concerning the initial values.

In contrast to Duan et al., Winkler works on a bounded convex domain with a smooth boundary. Moreover, he drops the smallness condition of the initial values by requiring more properties for the parameter functions. For sufficiently smooth, positive $\chi$ and sufficiently smooth $f$ with $f(s)>0$ for $s>0$ and $f(0)=0$, it is assumed

$$
\left(\frac{f}{\chi}\right)^{\prime}>0, \quad\left(\frac{f}{\chi}\right)^{\prime \prime} \leq 0, \quad(f \chi)^{\prime} \geq 0 \quad \text { on }[0, \infty)
$$

Theorem 1.1 (i) in [32] states the global classical solvability for (1) for sufficiently smooth (not necessarily small) initial data in spatial dimension two. In 3D he establishes global weak solvability for the related chemotaxis-Stokes equations, where the non-linear term $u \cdot \nabla u$ is neglected in the Navier-Stokes equations.

Recently, he extended this result by proving the existence of a global weak solution of (1) in dimension 3 for the full chemotaxis-Navier-Stokes equation under similar conditions (see [33]).

Chae et al. [4] show global (weak) large-data solution of (1) on the whole $\mathbb{R}^{N}$ for $N=2,3$ by mainly requiring $\varphi, \chi, f, \chi^{\prime}, f^{\prime} \geq 0$ and

$$
\sup |\chi(c)-\mu f(c)| \leq \varepsilon \quad \text { for all } c \geq 0
$$

for some $\mu \in \mathbb{R}$ and some $\varepsilon>0$ in spatial dimension two as well as $\varepsilon=0$ in spatial dimension three. In particular for $N=3$, we have $\chi=\mu f$.

Moreover, in the chemotaxis literature there is a branch, where the linear diffusion in the equation for $n$ is replaced by a porus-medium-like diffusion (see [3,8,34]). Here, [8] proves the global-in-time existence of a weak solution of (1) for large initial data, where its first equation is replaced by

$$
n_{t}+u \cdot \nabla n=\delta \Delta n^{m}-\nabla \cdot(n \chi(c) \nabla c), \quad x \in \mathbb{R}^{3}, t>0
$$

for any $m \geq 1$ and $\Omega=\mathbb{R}^{3}$.

The mentioned articles assume a total constant number of bacteria undermining death and reproduction. However, there has been a lot of progress in understanding the influence of the growth of the bacteria population for some related chemotaxis equations

$$
\left\{\begin{aligned}
n_{t} & =\Delta n-\chi \nabla \cdot(n \nabla c)+l(n), & & x \in \Omega, t>0, \\
\tau c_{t} & =\Delta c-n+c, & & x \in \Omega, t>0
\end{aligned}\right.
$$

for some $\tau>0$ and $\chi \in \mathbb{R}$ for some well-behaving $l$ (cf. [1,19,22,23]). In [31], Winkler investigates the influence of a logistic growth of the bacteria population. In his article, he proves the existence of a unique bounded global-in-time classical solution of (4) in a bounded convex domain $\Omega \subseteq \mathbb{R}^{N}$ for any $N \geq 1, \tau>0$ and $\chi \in \mathbb{R}$, where $l$ is a smooth function fulfilling

$$
l(x) \leq r x-d x^{2} \quad \text { for } x \geq 0
$$

for some $r>0$ and for sufficiently large $d>0$. Restricting to the two dimensional case and assuming $\Omega=\mathbb{R}^{2}$, it is possible to drop the largeness condition on $d$ without changing the result (see [22]).

Note that the system (4) does not involve an equation for a liquid, whereas in [10] the system (4) is supplemented by the Stokes equation and reads

$$
\left\{\begin{aligned}
n_{t}+u \cdot \nabla n & =\Delta n-\chi \nabla \cdot(n \nabla c)+l(n), & & x \in \Omega, t>0, \\
\tau c_{t}+u \cdot \nabla c & =\Delta c-n+c, & & x \in \Omega, t>0, \\
u_{t}+\nabla P & =\Delta u-n \nabla \varphi, & & x \in \Omega, t>0, \\
\nabla \cdot u & =0, & & x \in \Omega, t>0
\end{aligned}\right.
$$

for a given potential $\varphi$. Setting $l(n)=-\varepsilon n^{2}$ for some $\varepsilon>0$, Espejo and Suzuki prove in [10] the existence of a global weak solution of (4) in conjunction with (5) for $\Omega$ being either a smooth, bounded domain in $\mathbb{R}^{2}$ or the whole $\mathbb{R}^{2}$. Moreover, Winkler and Tao [36] extended this result using $l(n)=r n-\mu n^{2}$ for some $r \geq 0$ and $\mu>0$. For a bounded domain $\Omega \in \mathbb{R}^{2}$ with smooth boundary, they proved that (4) in combination with (5) admits a bounded classical 
solution requiring sufficiently smooth initial data. In addition, requiring $r=0$, the solution decays in time to zero in $L^{\infty}(\bar{\Omega})$ (cf. [36]).

It shall be emphasised that there are several related problems to the standard chemotaxis system: In [35], Winkler analyses a chemotaxis-Stokes system, where the equation concerning $n$ is replaced by

$$
n_{t}+u \cdot \nabla n=\Delta n-\nabla \cdot(n S(x, n, c) \cdot \nabla c) \quad x \in \Omega, t>0 .
$$

Assuming some smoothness properties, no flux boundary conditions as well as

$$
|S(x, n, c)| \leq S_{0}(c) \quad \text { for all }(x, n, c) \in \bar{\Omega} \times[0, \infty)^{2}
$$

for some nondecreasing $S_{0}:[0, \infty) \mapsto \mathbb{R}$, Winkler derives the existence of a global generalised solution, which is attracted by $\left(\bar{n}_{0}, c_{0}, u_{0}\right)$. Here, $\left(n_{0}, c_{0}, u_{0}\right)$ is the initial guess and $\bar{n}_{0}$ represents the average of $n_{0}$ with respect to $\Omega$.

Another branch of related problems supposes a nonlinear diffusion, i.e. exchanging $\Delta n$ by $\Delta n^{m}$ in equation (1) for some $m \geq 1$ (see $[7,26,27])$.

\subsection{Extension of the model}

The aim of this paper is to derive the global solvability of large-data solutions as in [4] and in [32]. We underline that the conditions (2) and (3) are very restrictive to the functions $\chi$ and $f$. Furthermore, a prototype case for the chemotaxis-Navier-Stokes equations, introduced by Winkler [32] by setting

$$
\nabla \varphi \equiv \text { const. }, \quad \chi \equiv \text { const. }, \quad f \equiv i d,
$$

clearly undermines the conditions (3) used in [4]. Although [32] treats this prototype case, the global existence theorem is restricted to the simplified chemotaxis-Stokes equations in spatial dimension three.

In order to facilitate the conditions of $\chi$ and $f$, we extend the model by assuming a logistic growth of the bacteria population and add the term $l(n)$ to the first equation, whose prototype is given by

$$
l(n):=\kappa n-\mu n^{2}, \quad \kappa, \mu>0 .
$$

Here $\kappa n$ models the reproduction of the bacteria which is proportional to their total number and $-\mu n^{2}$ describes a death rate, which prevents an excessive increase of the cell density.

In accord with [32], we study the chemotaxis-Navier-Stokes system in a bounded convex domain $\Omega$ with a smooth boundary. Let us assume that the system (1) describes the behaviour of oxygen-driven bacteria living in a water drop in which oxygen is solved. According to equation $(1)_{2}$ the oxygen is consumed by the bacteria and hence, the total mass of oxygen decreases over time. If the drop is surrounded by air, there exists a reversible reaction between the solved oxygen in the drop and the free oxygen in the air. Therefore, oxygen may diffuse into the drop, which may change the behaviour of the bacteria. Clearly, this effect takes place on the boundary of $\Omega$. Therefore, we require different boundary conditions comparing to [32]:

In case of an ideal solution, the behaviour of the oxygen exchange can be described by Raoult's law, which connects the rate of incoming oxygen to the partial vapour pressure of the oxygen in the surroundings. The origin of this law is given by Atkins and de Paula in terms of a molecular interpretation (see [2] section 5.3 on page 144). Fortunately, this suits our settings:

Atkins and de Paula explain that the leaving rate of the oxygen molecules is proportional to the total number of molecules on the surface. The condensing rate of the free oxygen-molecules depends proportionally on their partial pressure if we model an ideal solution. In case of a real solution, we have to drop the proportionality. Nevertheless, we will assume that the vapour pressure of the free oxygen is given and thereby, the incoming rate of oxygen is known. In conclusion, we set the normal derivative of the oxygen concentration on the boundary to

$$
\frac{\partial c}{\partial v}(x, t)=-a_{1}(x) c(x, t)+a_{2}(x, t), \quad(x, t) \in \partial \Omega \times(0, \infty)
$$

for some given functions $a_{1} \in C^{\infty}(\partial \Omega)$ and $a_{2} \in C^{\infty}(\partial \Omega \times[0, \infty))$. Here $-a_{1}(x) c(x, t)$ describes the outgoing oxygen-molecules; therefore, we assume that

$a_{1}(x)$ is positive for all $x \in \partial \Omega$. 
The second function $a_{2}$ models the incoming oxygen and depends on the known vapour pressure of the free oxygen. $a_{2}$ shall be positive or at least non-negative.

In view of the term $-\nabla \cdot(n \chi(c) \nabla c)$ in equation $(1)_{1}$, we also need to change the boundary conditions for $n$ unless we permit the bacteria to leave the drop. This is the reason for introducing the no-flux boundary conditions for $n$, namely

$$
\frac{\partial n}{\partial v}=n \chi(c) \frac{\partial c}{\partial v} \quad \text { on } \partial \Omega \times(0, \infty) .
$$

Up to this point, we have assumed that the exchange of oxygen only takes place at the boundary. But we may think of modelling a thin liquid film in 3D by analysing the equations in spatial dimension two. In this case, an oxygen exchange is reasonable everywhere in the film. We thus also introduce a sufficiently smooth function $h(x, t, c)$ modelling the oxygen exchange, which we add to the right-hand side of equation $(1)_{2}$. All in all we obtain the system

$$
\left\{\begin{aligned}
n_{t}+u \cdot \nabla n & =\Delta n-\nabla \cdot(n \chi(c) \nabla c)+l(n), & & x \in \Omega, t>0, \\
c_{t}+u \cdot \nabla c & =\Delta c-n f(c)+h(x, t, c), & & x \in \Omega, t>0, \\
u_{t} & =v \Delta u+u \cdot \nabla u+\nabla P-n \nabla \varphi, & & x \in \Omega, t>0, \\
\nabla \cdot u & =0, & & x \in \Omega, t>0
\end{aligned}\right.
$$

in conjunction with the initial data $(n, c, u)(\cdot, 0)=\left(n_{0}, c_{0}, u_{0}\right)$ and the boundary conditions (6) and (7).

\subsection{Main results}

Let $\Omega$ be a bounded domain in $\mathbb{R}^{N}$ with smooth boundary for $N=2,3$. We assume the following conditions for the standard parameter functions $\chi, f$ and $\varphi$ : Let $\gamma>0$, the functions $\chi \in C^{3+\gamma}(\mathbb{R})$ and $f \in C^{2+\gamma}(\mathbb{R})$ fulfil

$$
\chi \geq 0 \text { and } f(s) \geq 0, s \geq 0, f(0)=0 .
$$

Moreover, let $\varphi$ be differentiable such that $\nabla \varphi \in C^{\gamma}(\bar{\Omega})^{N}$ for some $\gamma>0$.

The property of $l(n)$ modelling a logistic growth is obtained by the assumption

$$
l(x) \leq r-d x^{2} \quad \text { for } x>0
$$

for some $r, d>0$. For technical reasons we also suppose that $l \in C^{2+\gamma}(\mathbb{R}), \gamma>0$, fulfils

$$
l(0) \geq 0 \text { and }|l(x)| \leq M+|x|^{Q} \text { for all } x \in \mathbb{R}
$$

and some $Q, M>0$. We fix the functions modelling the oxygen exchange by requiring $h \in C^{2+\gamma}(\bar{\Omega} \times[0, \infty) \times \mathbb{R})$ for some $\gamma>0$ and $a_{1} \in C^{\infty}(\partial \Omega), a_{2} \in C^{\infty}(\partial \Omega \times[0, \infty))$. As above, we assume that they are bounded and fulfil

$$
a_{1}(x)>0 \text { and } a_{2}(x, t) \geq 0,(x, t) \in \partial \Omega \times(0, \infty) .
$$

Moreover, we need

$$
h(\cdot, \cdot, c) \geq 0 \Leftrightarrow c \leq M
$$

for some $M>0$. In addition to these conditions for the parameter functions, we also require sufficient smoothness of the initial data:

$$
\left\{\begin{aligned}
0<n_{0} & \in C^{0}(\bar{\Omega}) \cap H^{1}(\Omega) \\
0<c_{0} & \in W^{1,10}(\Omega), \\
u_{0} & \in D\left(A^{\alpha}\right) \quad \text { for some } \frac{N}{4}<\alpha<1 .
\end{aligned}\right.
$$

Proposition 1.1. Assume that (8)-(13) hold. Then there exists a time $T^{*} \in(0, \infty]$ such that (cNS) admits a classical solution $(n, c, u, P)$ on $\bar{\Omega} \times\left[0, T^{*}\right)$ which is unique up to a time depending constant to the pressure $P$ in the class of 


$$
\left\{\begin{array}{l}
n \in C^{4+2 \mu, 2+\mu}(\bar{\Omega} \times(0, T)) \cap C^{0}(\bar{\Omega} \times[0, T)) \\
\quad \cap L^{\infty}\left(0, T ; W^{1,2}(\Omega)\right) \cap L^{\infty}\left(0, \infty ; L^{p}(\Omega)\right), \\
c \in C^{4+2 \mu, 2+\mu}(\bar{\Omega} \times(0, T)) \cap C^{0}(\bar{\Omega} \times[0, T)) \cap L^{\infty}\left(0, T ; W^{1,8}(\Omega)\right), \\
u \in C^{2+2 \mu, 1+\mu}(\bar{\Omega} \times(0, T)) \cap C^{0}(\bar{\Omega} \times[0, T)) \cap L^{\infty}\left(0, T ; D\left(A^{\alpha}\right)\right), \\
P \in C^{1+\mu, \mu}(\bar{\Omega} \times(0, T)) \cap L^{\infty}\left(0, T ; W^{1,2}(\Omega)\right)
\end{array}\right.
$$

for every $T<T^{*}, p<\infty$ and some $\mu>0$. In addition, the function $n$, c are positive.

Theorem 1.2 $(N=2)$. Let $\Omega$ be convex. The solution given by Proposition 1.1 is indeed global in time, i.e. $T^{*}=\infty$ and each $L^{q}(\Omega)$ norm of $n$ is globally bounded in time for every $q \geq 1$.

Theorem $1.3(N=3)$. Assume that (8)-(13) hold and that $\Omega$ is convex; then (cNS) admits at least a global weak solution in sense of Definition 1.5 below.

Remark 1.4. The convexity of $\Omega$ is assumed for technical reasons: It allows us to find an a priori estimate for $\nabla c$ in Lemma 3.9 in order to prevent a blow-up of the solution. This trick has already been used in [31]. It is not known to the author whether Theorem 1.2 and Theorem 1.3 are still correct without assuming the convexity of $\Omega$. However, since $\Omega$ describes a water drop, it is reasonable to suppose that it is convex.

Definition 1.5. Assume that

$$
\left\{\begin{array}{l}
n \in L_{l o c}^{2}\left(0, \infty ; L^{2}(\Omega)\right) \cap L_{l o c}^{\frac{4}{3}}\left(0, \infty ; W^{1, \frac{4}{3}}(\Omega)\right) \\
c \in L^{\infty}(\Omega \times(0, \infty)) \cap L_{l o c}^{2}\left(0, \infty ; W^{2,2}(\Omega)\right) \cap C^{0}\left([0, \infty) ; L^{2}(\Omega)\right) \\
u \in L^{\infty}\left(0, \infty ; L_{\sigma}^{2}(\Omega)\right) \cap L_{l o c}^{2}\left(0, \infty ; W_{\sigma}^{1,2}(\Omega)\right)
\end{array}\right.
$$

and $l(n) \in L^{1}(\Omega \times(0, t))$ as well as $c \in W^{1, \frac{4}{3}}(\Omega \times(0, t))$ are true for all $t>0$.

We call $(n, c, u)$ a global weak solution of $(\mathrm{cNS})$ if we have

$$
\begin{aligned}
& \iint_{\Omega} n \Psi_{1 t}+\iint_{0}^{\infty} n u \cdot \nabla \Psi_{1}+\iint_{0}^{\infty} \nabla n \cdot \nabla \Psi_{1} \\
& =\int_{\Omega} n_{0} \Psi_{1}(0, \cdot)-\iint_{0} n \chi(c) \nabla c \cdot \nabla \Psi_{1}+\iint_{0} l(n) \Psi_{1}, \\
& \iint_{\Omega}^{\infty} c \Psi_{2 t}+\iint_{0}^{\infty} c u \cdot \nabla \Psi_{2}-\iint_{0}^{\infty} \Delta c \Psi_{2} \\
& =\int_{\Omega} c_{0} \Psi_{2}(0, \cdot)-\iint_{0}^{\infty} c f(n) \Psi_{2}+\iint_{0}^{\infty} h(c) \Psi_{2}, \\
& \int_{0}^{\infty} \int_{\Omega} u \xi_{t}+\iint_{0}^{\infty} u \otimes u: \nabla \xi+v \iint_{0}^{\infty} \nabla u: \nabla \xi=\int_{\Omega} u_{0} \cdot \xi(0)+\iint_{0} n \nabla \varphi \cdot \xi
\end{aligned}
$$

for all $\Psi_{1}, \Psi_{2} \in C_{c}^{\infty}(\bar{\Omega} \times[0, \infty))$ and $\xi \in C_{c}^{\infty}(\bar{\Omega} \times[0, \infty))^{N}$ with $\nabla \cdot \xi=0$.

Proposition 1.6 (Strong solvability of the oxygen equation). There exists a weak solution of (cNS) obtained by Theorem 1.3 such that $(n, c, u)$ is a strong solution of $(\mathrm{cNS})_{2}$, i.e. $c_{t}, \nabla c$ and $\Delta c$ exist almost everywhere and belong to $L_{l o c}^{\frac{4}{3}}\left(0, \infty ; L^{\frac{4}{3}}(\Omega)\right)$ such that we have 


$$
c_{t}+u \cdot \nabla c=\Delta c-n f(c)+h(c) \quad \text { a.e. on } \Omega \times(0, \infty) .
$$

Furthermore, $c(\cdot, t) \rightarrow c_{0}$ in $L^{2}(\Omega)$ as $t \rightarrow 0$. In addition, $\frac{\partial c}{\partial v}$ and c exist almost everywhere in $\partial \Omega$ with respect to the surface measure of $\partial \Omega$ and they are elements of $L_{\text {loc }}^{2}\left(0, \infty ; L^{2}(\partial \Omega)\right)$. The function c fulfils the boundary conditions

$$
\frac{\partial c}{\partial v}=-a_{1} c+a_{2} \quad \text { a.e. on } \partial \Omega \times(0, \infty) .
$$

Lemma 1.7. Let $(u, c, n, P)$ be a solution of $(\mathrm{cNS})$. We have

$$
\sup _{0 \leq t<T_{\max }}\|n(t)\|_{L^{1}(\Omega)}<\infty
$$

and we deduce

$$
c(t) \leq \bar{c}:=\max \left\{M,\left\|\frac{a_{2}}{a_{1}}\right\|_{L^{\infty}\left(\partial \Omega \times \mathbb{R}_{+}\right)},\left\|c_{0}\right\|_{L^{\infty}(\Omega)}\right\}
$$

for all $0 \leq t<T_{\max }$, where $M:=\sup \{c \mid h(c)>0\}<\infty$.

\subsection{Overview and outlook}

The proof of the main theorems is mainly divided into three parts:

The succeeding section provides our first goal: the local existence of a unique classical solution of the chemotaxisNavier-Stokes equations. Dealing with technical difficulties due to the non-homogeneous boundary condition, Section 2 develops a transformation permitting the standard usage of semi-group theory. We state a local existence result which slightly generalises Proposition 1.1 and which introduces a blow-up criterion being crucial for further steps.

Section 3 develops a basic machinery of a priori estimates which are necessary for the last section in order to pass from a local to a global solvability. In addition, we develop the key estimate for the global in time boundedness of $\|n\|_{L^{q}(\Omega)}$ for all $q<\infty$.

Finally, we combine the blow-up criterion with the a priori estimates to earn global existence, which can be directly achieved in the two dimensional case. For $N=3$ we have get more involved and define an approximating sequence for the desired weak solution and cope with its convergence.

For a further research project, it would be interesting to learn more about the gained solution: Obviously, the standard chemotaxis-Navier-Stokes equations possess a trivial solution, i.e. $(n, c, u, P) \equiv 0$. In contrast to that, after the extension of the model, the boundary conditions for $c$ disagree with $c \equiv 0$ in the prototype case. Therefore, we may assume that the long-time behaviour for small-data solutions is different to that one proven in [9]. In addition, since the bacteria consume the solved oxygen, we may interpret the boundary as an oxygen source. Thereby, having in mind that the bacteria need oxygen to survive, it is reasonable to suppose that the bacteria are likely to accumulate at the boundary. This leads to the open question if there exists a time-independent distribution of $(n, c, u, P)$ which attracts appropriate small-data solutions of the extended chemotaxis-Navier-Stokes system.

\section{Local solution and transformation into homogeneous boundary conditions}

First of all, we note that according to [17] Theorem 9.4 and the Gagliardo-Nirenberg embedding properties, there exist bounded functions $g_{1} \in C^{\infty}(\bar{\Omega})$ and $g_{2} \in C^{\infty}(\bar{\Omega} \times[0, \infty))$ such that we have

$$
\frac{\partial g_{1}}{\partial v}(x)=-a_{1}(x), g_{2}(x, t)=\frac{a_{2}(x, t)}{a_{1}(x)} \text { with } \frac{\partial g_{2}}{\partial v}(x, t)=0,(x, t) \in \partial \Omega \times[0, \infty) .
$$

Since these functions are not unique, we now choose such $g_{1}$ and $g_{2}$ and fix them. In this and the following section, we continue using the expressions $g_{1}$ and $g_{2}$ for those two functions.

Remark 2.1. We can rewrite the boundary conditions for $c$ to

$$
\frac{\partial c}{\partial v}(x, t)=\frac{\partial g_{1}}{\partial v}(x)\left(c(x, t)-g_{2}(x, t)\right), \quad(x, t) \in \partial \Omega \times[0, \infty) .
$$


Here $\frac{\partial g_{1}}{\partial v}$ is negative on the boundary. We may interpret $g_{2}(x, t)$ as the critical oxygen concentration which decides whether oxygen condensates or vaporises at a point $x$ on the boundary at a time $t$. Clearly, we have $g_{2} \geq 0$ on $\partial \Omega$.

Since the system (cNS) has non-linear inhomogeneous boundary conditions, a standard semi-group argument cannot directly be applied. It has to be postponed after transforming the equations into homogeneous boundary conditions.

Definition 2.2. Let $n, c: \bar{\Omega} \times\left[0, T_{\max }\right) \rightarrow \mathbb{R}$ be a solution of (cNS); we define

$$
\begin{aligned}
\tilde{c} & :=e^{-g_{1}}\left(c-g_{2}\right), \\
\tilde{n} & :=n A(c)=n A\left(e^{g_{1}} \tilde{c}+g_{2}\right), \\
A(c) & :=\exp \left(-\int_{0}^{c} \chi(s) d s\right)
\end{aligned}
$$

having in mind

$$
\frac{\partial \tilde{n}}{\partial v}=\frac{\partial \tilde{c}}{\partial v}=0 \quad \text { on } \partial \Omega
$$

In addition we also transform the initial guess to

$$
\tilde{n}_{0}:=n_{0} A\left(c_{0}\right) \quad \text { and } \quad \tilde{c}_{0}:=e^{-g_{1}}\left(c_{0}-g_{2}(\cdot, 0)\right) .
$$

The strategy to derive the solvability of (cNS) consists of solving a transformed PDE for the parameters $\tilde{n}, \tilde{c}, u$. Therefore, we are also interested in a backward transformation which defines $n, c$ by using $\tilde{n}, \tilde{c}$ :

Definition 2.3. We define the backward transformations $T_{n}^{x, t}: \mathbb{R}^{2} \rightarrow \mathbb{R}$ and $T_{c}^{x, t}: \mathbb{R} \rightarrow \mathbb{R}$ via

$$
T_{c}^{x, t}(b)=e^{g_{1}(x)} b+g_{2}(x, t), \quad T_{n}^{x, t}(a, b)=a A\left(-T_{c}^{x, t}(a, b)\right),
$$

for $x \in \bar{\Omega}$ and $0 \leq t<\infty$. Now let $\varphi_{1}, \varphi_{2}: \bar{\Omega} \times\left[0, T_{\max }\right) \rightarrow \mathbb{R}$ be two functions for some $T_{\max }>0$. Then we put

$$
\left(\begin{array}{c}
T_{n}\left(\varphi_{1}, \varphi_{2}\right) \\
T_{c}\left(\varphi_{2}\right)
\end{array}\right)(x, t):=\left(\begin{array}{c}
T_{n}^{x, t}\left(\varphi_{1}(x, t), \varphi_{2}(x, t)\right) \\
T_{c}^{x, t}\left(\varphi_{2}(x, t)\right)
\end{array}\right)
$$

for all $x \in \bar{\Omega}$ and $0 \leq t<T_{\max }$.

Remark 2.4. Clearly, we have

$$
n=T_{n}(\tilde{n}, \tilde{c}) \quad \text { and } \quad c=T_{c}(\tilde{c}) .
$$

Lemma 2.5. There exist functions $\tilde{R} \in C^{2+\mu}\left(\bar{\Omega} \times \mathbb{R}_{+} \times \mathbb{R}^{2+N}\right)$, $\tilde{l} \in C^{2+\mu}\left(\bar{\Omega} \times \mathbb{R}_{+} \times \mathbb{R}^{2}\right)$ and $\tilde{h} \in C^{2+\mu}\left(\bar{\Omega} \times \mathbb{R}_{+} \times\right.$ $\left.\mathbb{R}^{2+2 N}\right)$ for some $\mu>0$ such that $(n, c, u, P)$ solves $(\mathrm{cNS})_{1}$ and $(\mathrm{cNS})_{2}$ iff $(\tilde{n}, \tilde{c}, u, P)$ is a solution of

$$
\begin{gathered}
\tilde{n}_{t}+u \cdot \nabla \tilde{n}=\Delta \tilde{n}-2 \tilde{\mathbf{a}}(\tilde{c}, \nabla \tilde{c}) \cdot \nabla \tilde{n}+\nabla \cdot(\tilde{n} \tilde{\mathbf{a}}(\tilde{c}, \nabla \tilde{c})) \\
+\tilde{R}(\tilde{n}, \tilde{c}, \nabla \tilde{c})+\tilde{l}(\tilde{n}, \tilde{c})
\end{gathered}
$$

and

$$
\tilde{c}_{t}+u \cdot \nabla \tilde{c}=\Delta \tilde{c}+\tilde{h}(\tilde{n}, \tilde{c}, \nabla \tilde{c}, u),
$$

where we have dropped the $x, t$ dependency in the notation of the equations. In addition, the functions $\tilde{R}, \tilde{h}$ can be written in use of some differentiable auxiliary functions $F_{1}, \boldsymbol{F}_{2}, \ldots$ as

$$
\begin{aligned}
\tilde{\mathbf{a}}(\beta, v) & =F_{1}(\beta) v+\boldsymbol{F}_{2}(\beta) \\
\tilde{R}(\alpha, \beta, v) & =F_{3}(\alpha, \beta)\left|v+\boldsymbol{F}_{4}(\beta)\right|^{2}+F_{5}(\alpha, \beta) \\
\tilde{l}(\alpha, \beta) & =F_{6}(\beta) l\left(\alpha F_{7}(\beta)\right) \\
\tilde{h}(x, t, \alpha, \beta, v, w) & =\boldsymbol{F}_{8}(\beta) \cdot w+\boldsymbol{F}_{9} \cdot v+F_{10}(\alpha, \beta),
\end{aligned}
$$


for $\alpha, \beta \in \mathbb{R}, w, v \in \mathbb{R}^{N}$, where we have again neglected the dependency on $(x, t) \in \bar{\Omega} \times(0, \infty)$. Here, a bold letter denotes a vector valued function. We have the estimate

$$
\left|F_{i}(\alpha, \beta)\right| \leq(1+|\alpha|) C_{\beta} \quad \text { and } \quad\left|\mathbf{F}_{i}(\beta)\right| \leq C_{\beta}
$$

for all $\alpha, \beta, i$, whenever $F_{i}$ is involving on $\alpha$, where $C_{\beta}$ is depending on $\beta$. To be more precise, $\boldsymbol{F}_{9}$ does not depend on $\beta$ and hence it is globally bounded.

Proof. This lemma can be shown by a direct and excessive calculation which is left to the reader. However, the auxiliary functions are given by

$$
\begin{gathered}
\tilde{\mathbf{a}}: \bar{\Omega} \times \mathbb{R}_{+} \times \mathbb{R} \times \mathbb{R}^{N} \rightarrow \mathbb{R}^{N} \\
(x, t, s, v) \mapsto-\chi\left(T_{c}^{x, t}(s)\right)\left(v+s \nabla g_{1}(x)+\nabla g_{2}(x, t) e^{-g_{1}(x)}\right) e^{g_{1}(x)}
\end{gathered}
$$

and

$$
\tilde{l}\left(\psi_{1}, \psi_{2}\right):=A\left(T_{c}\left(\psi_{2}\right)\right) l\left(T_{n}\left(\psi_{1}, \psi_{2}\right)\right)
$$

for $\psi_{1}, \psi_{2}$ being real valued functions on $\bar{\Omega} \times[0, T)$ for some $T>0$. Moreover, we have

$$
\begin{aligned}
& R\left(x, t, s_{1}, s_{2}, v\right):=-s_{1} \chi^{\prime}\left(s_{2}\right)|v|^{2}+s_{1}^{2} \chi\left(s_{2}\right) \frac{f\left(s_{2}\right)}{A\left(s_{2}\right)}-s_{1} \chi\left(s_{2}\right) h\left(x, t, s_{2}\right), \\
& \left.\tilde{R}\left(x, t, s_{1}, s_{2}, v\right):=R\left(x, t, s_{1}, T_{c}^{x, t}\left(s_{2}\right), v e^{g_{1}(x)}\right)+s_{2} \nabla e^{g_{1}(x)}+\nabla g_{2}(x, t)\right)
\end{aligned}
$$

for $x \in \bar{\Omega}, t>0, s_{1}, s_{2} \in \mathbb{R}$ and $v \in \mathbb{R}^{N}$ as well as

$$
\begin{aligned}
\tilde{h}\left(x, t, s_{1}, s_{2}, v, w\right):= & -w \cdot\left(s_{2} \nabla g_{1}(x)+\nabla g_{2}(x, t) e^{-g_{1}(x)}\right) \\
& +2 v \cdot \nabla g_{1}(x)+s_{2}\left(\left|\nabla g_{1}(x)\right|^{2}+\Delta g_{1}(x)\right)+\Delta g_{2}(x, t) e^{-g_{1}(x)} \\
& +e^{-g_{1}(x)}\left(-T_{n}^{x, t}\left(s_{1}, s_{2}\right) f\left(T_{c}^{x, t}\left(s_{2}\right)\right)+h\left(x, t, T_{c}^{x, t}\left(s_{2}\right)-\partial_{t} g_{2}(x, t)\right)\right.
\end{aligned}
$$

for $x \in \bar{\Omega}, t>0, s_{1}, s_{2} \in \mathbb{R}$ and $v, w \in \mathbb{R}^{N}$.

As a matter of prudence, we approximate the Navier-Stokes equations in our system in order to obtain a weak solution in spatial dimension three. Thus, the (approximate) homogeneous chemotaxis-Navier-Stokes equations are given by:

$$
\left\{\begin{aligned}
\tilde{n}_{t}+u \cdot \nabla \tilde{n} & =\Delta \tilde{n}-2 \tilde{\mathbf{a}}(\tilde{c}, \nabla \tilde{c}) \cdot \nabla \tilde{n}+\nabla \cdot(\tilde{n} \tilde{\mathbf{a}}(\tilde{c}, \nabla \tilde{c})) & \\
& +\tilde{R}(\tilde{n}, \tilde{c}, \nabla \tilde{c}, u)+\tilde{l}(\tilde{n}, \tilde{c}), & x \in \Omega, t>0, \\
\tilde{c}_{t}+u \cdot \nabla \tilde{c} & =\Delta \tilde{c}+\tilde{h}(\tilde{n}, \tilde{c}, \nabla \tilde{c}, u), & x \in \Omega, t>0, \\
u^{\prime}(t) & =A u+P^{m} B(u(t), u(t))-P^{m}\left(T_{n}(\tilde{n}, \tilde{c}) \nabla \varphi\right) & \text { in } L_{\sigma}^{2}(\Omega), t>0, \\
(\tilde{n}, \tilde{c}, u)(x, 0) & =\left(\tilde{n}_{0}, \tilde{c}_{0}, P^{m} u_{0}\right)(x), & x \in \Omega, \\
\frac{\partial \tilde{n}}{\partial v} & =0, \frac{\partial \tilde{c}}{\partial v}=0 & \text { on } \partial \Omega .
\end{aligned}\right.
$$

Here, we write $A=-\mathcal{P} \Delta$, where $\mathcal{P}$ is the Leray projection. We define $P^{m}$ for $m \in \mathbb{N} \cup\{\infty\}$ as the projection onto the first $m$ eigenspaces of $A$ and $B(u, v)$ denotes the bilinear form $u \cdot \nabla v$. Note that we also allow $m=\infty$, in which the equation for $u$ turns into the common Navier-Stokes equations.

The following proposition corresponds to Lemma 2.1 in [32]. Unlike the situation in [32], we consider a right-hand side of equation (25) 1 with some extra term like $\nabla n \cdot \tilde{\mathbf{a}}(\tilde{c}, \nabla \tilde{c})$. Unfortunately, $\nabla n \cdot \tilde{\mathbf{a}}(\tilde{c}, \nabla \tilde{c})$ is not in divergence form. Treating these additional terms turns out to be slightly more involved than it is in [32]. Because of this, we change Lemma 2.1 in [32] to 
Proposition 2.6. Let $N \in\{2,3\}$ and $\Omega \subset \mathbb{R}^{N}$ be a bounded domain with smooth boundary.

Then there exists a maximal $T_{\max } \in(0, \infty]$ and $\mu>0$ such that (25) possesses a classical solution fulfilling for every $0<T<T_{\max }$

$$
(\tilde{n}, \tilde{c}, u) \in X_{T}:=L^{\infty}\left([0, T) ; L^{\infty}(\Omega) \cap H^{1}(\Omega) \times W^{1,4}(\Omega) \times D\left(A^{\alpha}\right)\right)
$$

such that

$$
\begin{aligned}
n, c & \in C^{2+2 \mu, 1+\mu}\left(\bar{\Omega} \times\left(0, T_{\max }\right)\right) \cap C^{0}\left(\bar{\Omega} \times\left[0, T_{\max }\right)\right) \\
u & \in C^{1+\mu}\left(0, T_{\max } ; L_{\sigma}^{2}(\Omega)\right) \cap C\left(\left[0, T_{\max }\right) ; L_{\sigma}^{2}(\Omega)\right) \cap C^{\mu}\left(\left[0, T_{\max }\right) ; D(A)\right) .
\end{aligned}
$$

Furthermore, if $T_{\max }$ is finite, we have the blow-up criterion

$$
\|\tilde{n}(t)\|_{L^{\infty}}+\|\nabla \tilde{n}(t)\|_{L^{2}}+\|\tilde{c}(t)\|_{W^{1,4}}+\left\|A^{\alpha} u(t)\right\|_{L^{2}} \rightarrow \infty \text { as } t \nearrow T_{\max } .
$$

Moreover, let $m=\infty$; then we have $u \in C^{2+2 \mu, 1+\mu}(\bar{\Omega} \times(0, T))$ for some $\mu>0$. In addition, there exists a function $P \in C^{1+\mu, \mu}(\Omega \times(\varepsilon, t))$ such that $(\tilde{n}, \tilde{c}, u, P)$ solve

$$
\left\{\begin{aligned}
u_{t}+(u \cdot \nabla) u & =v \Delta u+\nabla P+T_{n}(\tilde{n}, \tilde{c}) \nabla \varphi, & & \text { on } \Omega \times(0, T), \\
u & =0 & & \text { on } \partial \Omega \times(0, T), \\
u(\cdot, 0) & =u_{0}, & & \text { on } \Omega .
\end{aligned}\right.
$$

The function $P$ is unique up to a constant.

The proof of Proposition 2.6 begins with achieving a mild solvability of the equations by an application of Banach's fixed-point theorem similarly to [32].

Let $X_{T}$ be as in Proposition 2.6 and let $B_{R}$ denote the closed ball in $X_{T}$ centred at 0 with radius $R>0$. In order to utilise Banach's fixed-point theorem, we require an adequate mapping

$$
\Phi:=\left(\Phi_{1}, \Phi_{2}, \Phi_{3}\right): B_{R} \rightarrow X_{T},
$$

which needs to be a contraction. We define $\Phi(n, c, u)$ for $(n, c, u) \in B_{R}$ by putting

$$
\begin{aligned}
& \Phi_{1}(n, c, u)(t):=e^{t \Delta} \tilde{n}_{0}+\int_{0}^{t} e^{(t-s) \Delta}\left\{-2 \tilde{\mathbf{a}}\left(c, \nabla \Phi_{2}\right) \cdot \nabla n+\nabla \cdot(\tilde{n} \tilde{\mathbf{a}}-u \tilde{n})\right. \\
& \left.+\tilde{n} \tilde{R}\left(n, c, \nabla \Phi_{2}\right)+\tilde{l}(n, c)\right\}(s) d s, \\
& \Phi_{2}(n, c, u)(t):=e^{t \Delta} \tilde{c}_{0}-\int_{0}^{t} e^{(t-s) \Delta}\{-u \cdot \nabla c+\tilde{h}(n, c, \nabla c, u)\}(s) d s, \\
& \Phi_{3}(n, c, u)(t):=e^{-t A} u_{0}-\int_{0}^{t} e^{-(t-s) A} P^{m}\left\{u \cdot \nabla u-T_{n}(n, c) \nabla \varphi\right\}(s) d s .
\end{aligned}
$$

Here, $\left(e^{t \Delta}\right)_{t \geq 0}$ and $\left(e^{-t A}\right)_{t \geq 0}$ denote the Neumann heat semi-group and Stokes semi-groups with Dirichlet boundary conditions, respectively. The proof that $\Phi$ defines a contraction is analogue to the proof found in [32] and will be stated for the reader's convenience. Let us denote $B:=-\Delta+1$ as the sectorial operator with Neumann boundary conditions.

Remark 2.7. Note that the definition of $\Phi_{1}$ depends on $\Phi_{2}$. Replacing $\Phi_{2}$ by $c$ in the definition of (27) would cause more difficulties in showing a bound for $\Phi_{1}$. Moreover, it is no longer guaranteed that the mapping $\Phi$ is well-defined. Thus, the strategy to achieve the bound for $\Phi_{1}$ is to obtain an admissible regularity of $\Phi_{2}$ first.

(i) Let us prove that $\Phi_{2}$ is bounded in $W^{1,10}(\Omega)$. Applying the contractivity of the heat semi-group and the Theorems 1.6.1 and 1.4.1 in [14], we infer choosing $\alpha_{1}<1$ with $1-\frac{N}{10}<2 \alpha_{1}-\frac{N}{4}$ that 


$$
\begin{aligned}
& \left\|\Phi_{2}(n, c, u)(\cdot, t)\right\|_{W^{1,10}(\Omega)} \\
& \quad \leq\left\|e^{t \Delta} c_{0}\right\|_{W^{1,10}(\Omega)}+\int_{0}^{t}\left\|e^{-(t-s)(B-1)}(u(s) \cdot \nabla c(s)+\tilde{h})\right\|_{W^{1,10}(\Omega)} d s \\
& \quad \leq\left\|c_{0}\right\|_{W^{1,10}(\Omega)}+C_{2} \int_{0}^{t}(t-s)^{-\alpha_{1}}\|u(s) \cdot \nabla c(s)+\tilde{h}\|_{L^{4}(\Omega)} d s \\
& \quad \leq\left\|c_{0}\right\|_{W^{1,10}(\Omega)}+\frac{C(R)}{1-\alpha_{1}} T^{1-\alpha_{1}}
\end{aligned}
$$

holds for all $0<t<T$, where we used that $\tilde{h}$ is affine in $\nabla c$ and $\nabla c \in L^{\infty}\left(0, T ; L^{4}(\Omega)\right)$ as well as $u \in D\left(A^{\alpha}\right) \hookrightarrow$ $C^{0}(\Omega)$ since $\alpha>N / 4[25]$.

(ii) Note that we have $\nabla \cdot(n u)=u \cdot \nabla n+n \nabla \cdot u=u \cdot \nabla n$. An analogue argumentation as in (i) in conjunction with Lemma 1.3 (iv) [30] yields

$$
\begin{aligned}
& \|\Phi(n, c, u)(t)\|_{L^{\infty} \cap H^{1}} \\
& \leq\left\|e^{t \Delta} n_{0}\right\|_{L^{\infty} \cap H^{1}}+\int_{0}^{t}\left\|B^{\frac{10}{11}} e^{-(t-s)(B-1)}\left(2 \tilde{\mathbf{a}}\left(c, \nabla \Phi_{2}\right) \cdot \nabla n-\tilde{n} \tilde{R}-\tilde{l}\right)\right\|_{L^{\frac{5}{3}}} d s \\
& \quad+\int_{0}^{t}\left\|B^{\frac{2}{5}} e^{-(t-s)(B-1)}\right\|_{L^{4} \rightarrow L^{4}}\left\|e^{(t-s) \Delta} \nabla \cdot\left(n \tilde{\mathbf{a}}\left(c, \nabla \Phi_{2}\right)-u n\right)\right\|_{L^{4}} d s \\
& \leq\left\|n_{0}\right\|_{L^{\infty} \cap H^{1}}+\int_{0}^{t}(t-s)^{\frac{10}{11}}\left\|2 \tilde{\mathbf{a}}\left(c, \nabla \Phi_{2}\right) \cdot \nabla n-\tilde{n} \tilde{R}-\tilde{l}\right\|_{L^{\frac{5}{3}}} d s \\
& \quad+\int_{0}^{t}(t-s)^{\frac{9}{10}}\left\|n \tilde{\mathbf{a}}\left(c, \nabla \Phi_{2}\right)-u n\right\|_{L^{4}} d s \\
& \leq\left\|n_{0}\right\|_{L^{\infty} \cap H^{1}}+C(R) T \frac{1}{11},
\end{aligned}
$$

where we also used Hölder's inequality, the linear or quadratic bounds of $\tilde{\mathbf{a}}, \tilde{n}, \tilde{R}, \tilde{h}, \tilde{l}$ with respect to $\nabla c, u$ or $\nabla \Phi_{1}$ and the fact that $\nabla \Phi_{1}$ is locally bounded in $L^{10}(\Omega)$ according to (i).

(iii) Proceeding similarly, we infer

$$
\left\|\Phi_{3}(n, c, u)(t)\right\|_{D\left(A^{\alpha}\right)} \leq\left\|u_{0}\right\|_{D\left(A^{\alpha}\right)}+C_{19}(R) \cdot T^{1-\alpha} .
$$

Concluding we have seen, that $\Phi$ is bounded. We can also obtain a Lipschitz estimate with a Lipschitz constant $C(R) T^{\beta}$ for some $\beta, C(R)>0$ by the same procedure as above and therefore, we leave it to the reader.

Setting $R:=2\left\|\left(n_{0}, c_{0}, u_{0}\right)\right\|$, it clearly results from the Banach's fixed-point theorem that if $T$ is sufficiently small (depending only on $R$ ), then the mapping $\Phi: B_{R} \rightarrow B_{R}$ is a well-defined contraction and admits a unique fixed-point $(\tilde{n}, \tilde{c}, u) \in B_{R}$.

Having found a so-called mild solution of the chemotaxis-Navier-Stokes equation, a bootstrap argument using the regularity of the Stokes semi-group, the heat semi-group and further regularities for parabolic entails the desired regularity, cf. $[5,12,13,15,20,25]$.

Positivity: Note that we have $f(0)=0$ and $h(\cdot, \cdot, s) \geq 0$ for $s \leq 0$ as well as $\frac{\partial c}{\partial v}(x, t) \geq 0$ whenever $c(x, t) \leq 0$ for $x \in \partial \Omega, t>0$. Thus, a standard argument involving the $L^{2}$ norm of the negative part of $n$ and $c$ ensures that $n$ and $c$ are non-negative. The strictly positivity of $n$ and $c$ can be obtained by a second step using the strong maximum principle and the regularity of the solutions.

Uniqueness: Proving uniqueness with respect to the desired class, we assume that $(\tilde{n}, \tilde{c}, u)$ and $\left(\tilde{n}^{\prime}, \tilde{c}^{\prime}, u^{\prime}\right)$ are solutions in $X_{T}$ of (25) and fulfil the condition $\left.\|(\tilde{n}, \tilde{c}, u)\right)_{X_{T}} \| \leq R$ as well as $\left\|\left(\tilde{n}^{\prime}, \tilde{c}^{\prime}, u^{\prime}\right)\right\|_{X_{T}} \leq R$ for some $R>0$. 
Let $n, n^{\prime}, c, c^{\prime}$ be the backward transforms of $\tilde{n}, \tilde{n}^{\prime}, \tilde{c}, \tilde{c}^{\prime}$, respectively. Thus, $(n, c)$ and $\left(n^{\prime}, c^{\prime}\right)$ solve the equations (cNS) with the corresponding $u, u^{\prime}$ respectively. Likewise to [32], we multiply difference of the equations of $c$ and $c^{\prime}$ to obtain

$$
\begin{aligned}
\frac{d}{d t} \int_{\Omega}\left(c-c^{\prime}\right)^{2}+ & \int_{\Omega}\left|\nabla\left(c-c^{\prime}\right)\right|^{2} \\
= & +\int_{\Omega}(-n f(c)+h(c))\left(c-c^{\prime}\right)+\int_{\Omega}\left(n^{\prime} f\left(c^{\prime}\right)-h\left(c^{\prime}\right)\right)\left(c-c^{\prime}\right) \\
& -\int_{\Omega}\left(u \nabla c-u^{\prime} \nabla c^{\prime}\right)\left(c-c^{\prime}\right)+\int_{\partial \Omega}\left(a_{1}\left(c-c^{\prime}\right)+a_{2}\right)\left(c-c^{\prime}\right) .
\end{aligned}
$$

Note that the boundary term can be controlled with the aid of the $W^{1,2}(\Omega)$ norm of $c-c^{\prime}$. A similar treatment to the bacteria equation yields

$$
\begin{aligned}
\frac{d}{d t} \int_{\Omega}\left(n-n^{\prime}\right)^{2}+ & \int_{\Omega}\left|\nabla\left(n-n^{\prime}\right)\right|^{2} \\
= & +\int_{\Omega} n \chi(c) \nabla c \cdot \nabla\left(n-n^{\prime}\right)-\int_{\Omega} n^{\prime} \chi\left(c^{\prime}\right) \nabla c^{\prime} \cdot \nabla\left(n-n^{\prime}\right) \\
& -\int_{\Omega}\left(u \nabla n-u^{\prime} \nabla n^{\prime}\right)\left(n-n^{\prime}\right)+\int_{\Omega}\left(l(n)-l\left(n^{\prime}\right)\right)\left(n-n^{\prime}\right),
\end{aligned}
$$

where the boundary terms vanish. We can use exact the same argumentation as in [32] to see that $y(t):=\int_{\Omega}(n-$ $\left.n^{\prime}\right)^{2}+c_{1} \int_{\Omega}\left(n-n^{\prime}\right)^{2}+c_{2} \int_{\Omega}\left(n-n^{\prime}\right)^{2}$ satisfies $y^{\prime} \leq c_{2} y$ on some small time interval and for some positive constants $c_{1}, c_{2}, c_{3}$ which only depend on $\left.\|(n, c, u)\right)_{X_{T}}\|\|,\left(n^{\prime}, c^{\prime}, u^{\prime}\right) \|_{X_{T}}$. Note that these norms can directly be estimated using $\|(\tilde{n}, \tilde{c}, u))_{X_{T}}\|\|,\left(\tilde{n}^{\prime}, \tilde{c}^{\prime}, u^{\prime}\right) \|_{X_{T}} \leq R$. We infer $y(t)=0$ according to $y(0)=0$, which ensures the uniqueness of the solution.

\section{A priori estimates}

Throughout this section we always assume that $(\tilde{n}, \tilde{c}, u)$ is a classical local solution of (25) and $n, c$ denote the backward transforms of $\tilde{n}$ and $\tilde{c}$, restrictively, by means of Definition 2.2. Thus, requiring $m=\infty,(n, c, u)$ solve equations (cNS) for an adequate $P$.

\section{1. $n \log n$ energy estimate}

The first and main a priori estimate is a rather weak one. However, it possesses the advantage that the energy type estimate only slightly depends on the logistic growth since we will only use the smoothness and the following property of $l$ appearing in $(\mathrm{cNS})_{1}$ :

$$
l(n) \leq r-d n^{2} \text { for } n \geq 0 .
$$

By means of (33), we may change $l$ by subtracting a smooth non-negative function without changing the inequalities and the constants provided in this section. For example,

$$
l_{0}(n):=l(n) \quad \text { and } \quad l_{\varepsilon}(n):=l(n)-\varepsilon n^{3} \quad \text { for } n \geq 0
$$

admit the same $n \log n$ estimate for all $\varepsilon>0$. This estimate, which is uniform in $\varepsilon$, permits defining an adequate approximating solution of $(\mathrm{cNS})_{1}$ and hence plays a crucial role in gaining the weak solution in spatial dimension three.

In contrast to the $n \log n$ estimate in [32], we shift $n$ by Euler's number $e$ and work on an $(n+e) \log (n+e)$ argument since we do not want to waste much thought on the singularity of the logarithm. The first approach only uses the equation of $n$ : 
Lemma 3.1. There exist some constants $C_{1}, C_{2}>0$ such that

$$
\begin{aligned}
& \frac{d}{d t} \int_{\Omega}(n+e)(\log (n+e)-1)+\frac{1}{2} \int_{\Omega} \frac{|\nabla n|^{2}}{n+e}+\frac{d}{4} \int_{\Omega} n^{2} \\
& \quad \leq C_{1} \int_{\Omega} \chi(c)^{2}|\nabla c|^{4}+\frac{1}{2} \int_{\Omega}(l(n)-r) \log (n+e)+C_{2} .
\end{aligned}
$$

Proof. A direct differentiation and an integration by parts yield

$$
\begin{aligned}
\frac{d}{d t} \int_{\Omega}( & n+e)(\log (n+e)-1)-\int_{\Omega} l(n) \log (n+e) \\
= & \int_{\Omega} \Delta n \log (n+e)-\int_{\Omega} \nabla \cdot(n \chi(c) \nabla c) \log (n+e) \\
= & -\int_{\Omega} \frac{|\nabla n|^{2}}{n+e}+\int_{\Omega} \frac{n \chi(c)}{n+e} \nabla n \cdot \nabla c
\end{aligned}
$$

since the boundary integral vanishes and $\nabla \cdot u=0$. By Young's inequality, we may cut the unsigned term containing $\nabla c \cdot \nabla n$ into two parts to digest the part involving $\nabla n$ by the first integral on the right-hand side of the previous calculation. This leads to

$$
2 \int_{\Omega} \frac{n \chi(c)}{n+e} \nabla n \cdot \nabla c \leq \int_{\Omega} \frac{|\nabla n|^{2}}{n+e}+\int_{\Omega} \frac{n}{n+e} \chi(c)^{2} n|\nabla c|^{2} .
$$

Now we split the integral involving $\nabla c$ to clear the dependency on $n$ by use of Young's inequality. In addition, we observe $n \leq n+e$. Collecting these preparatory considerations, we estimate

$$
\frac{d}{d t} \int_{\Omega}(n+e)(\log (n+e)-1)+\frac{1}{2} \int_{\Omega} \frac{|\nabla n|^{2}}{n+e} \leq \frac{d}{8} \int_{\Omega} n^{2}+\frac{1}{2 d} \int_{\Omega} \chi(c)^{2}|\nabla c|^{4}+\int_{\Omega} l(n) \log (n+e) .
$$

Fortunately, the logistic growth fulfils $l(n) \leq r-d n^{2}$; we thus find a constant $C_{1}>0$ such that

$$
\frac{3 d}{8} \int_{\Omega} n^{2}+\frac{1}{2} \int_{\Omega}(l(n)+r) \log (n+e) \leq C_{1} .
$$

Note that we have inserted an additional integral of the logarithm of $n+e$ which is also bounded by $-l(n)$. Combining this with the previous inequality shows the assertion by noting that $c$ and in particular $\chi(c)$ is bounded according to Lemma 1.7.

In order to absorb the integral containing $|\nabla c|^{4}$, we make use of the second equation:

Lemma 3.2. There exist constants $C_{0}, C_{1}, C_{2}, C_{3}>0$ such that we have

$$
\begin{aligned}
\frac{1}{2} \frac{d}{d t} \int_{\Omega}|\nabla \tilde{c}|^{2} & +C_{0} \int_{\Omega}|\nabla \tilde{c}|^{4}+C_{1} \int_{\Omega}|\nabla \tilde{c}|^{2} \\
& \leq C_{2} \int_{\Omega}|\nabla u|^{2}+C_{3}\left(1+\int_{\Omega}|u|^{2}+\int_{\Omega} n^{2}\right) .
\end{aligned}
$$

Proof. Let us recall

$$
\tilde{h}(n, \tilde{c}, \nabla \tilde{c}, u)=\mathbf{F}_{8}(\tilde{c}) \cdot u+\mathbf{F}_{9} \cdot \nabla \tilde{c}+F_{10}(n, \tilde{c})
$$


where $\mathbf{F}_{8}, \mathbf{F}_{9}$ and $F_{10}$ are given by Lemma 2.5 and fulfil (24). Thus, with the aid of the inequalities in (24), we estimate $\tilde{h}^{2} \leq C\left(1+|u|^{2}+n^{2}+|\nabla \tilde{c}|^{2}\right)$ for some $C>0$. Testing the equation $\tilde{c}_{t}+u \cdot \nabla \tilde{c}=\Delta \tilde{c}+\tilde{h}(n, \tilde{c}, \nabla \tilde{c}, u)$ with $\Delta \tilde{c}$, we derive

$$
\frac{1}{2} \frac{d}{d t} \int_{\Omega}|\nabla \tilde{c}|^{2}+\int_{\Omega}\left|D^{2} \tilde{c}\right|^{2}=-\int_{\Omega} \Delta \tilde{c} \tilde{c}_{t}+\int_{\Omega}|\Delta \tilde{c}|^{2}=+\int_{\Omega} \Delta \tilde{c} u \cdot \nabla \tilde{c}-\int_{\Omega} \Delta c \tilde{h}
$$

since $\int_{\Omega}\left|D^{2} \tilde{c}\right|^{2}=\int_{\Omega}|\Delta \tilde{c}|^{2}$. First, we estimate the latter integral using Young's inequality

$$
-\int_{\Omega} \Delta \tilde{c} \tilde{h} \leq \frac{1}{4 N} \int_{\Omega}|\Delta \tilde{c}|^{2}+N \int_{\Omega} \tilde{h}^{2} \leq \frac{1}{4} \int_{\Omega}\left|D^{2} \tilde{c}\right|^{2}+N \int_{\Omega} \tilde{h}^{2} .
$$

For the second integral on the right-hand side, we note that $\nabla \cdot u=0$ implies $u \cdot \nabla \tilde{c}=\nabla \cdot(u \tilde{c})$. Since $u$ vanishes on the boundary, integration by parts ensures

$$
\begin{aligned}
-\int_{\Omega} \Delta \tilde{c} u \cdot \nabla \tilde{c} & =-\sum_{i, j} \int_{\Omega} \partial_{i i} \tilde{c} \partial_{j}\left((u)_{j} \tilde{c}\right) \\
& =-\sum_{i, j} \int_{\Omega} \partial_{i j} \tilde{c} \partial_{i}(u)_{j} \tilde{c}-\sum_{i, j} \int_{\Omega} \partial_{i j} \tilde{c}(u)_{j} \partial_{i} \tilde{c} \\
& =-\int_{\Omega} \tilde{c} D^{2} \tilde{c}: \nabla u-\sum_{i, j} \int_{\partial \Omega}\left(\partial_{i} \tilde{c}\right)^{2} \underbrace{(u)_{j}}_{=0}+\sum_{i} \int_{\Omega}\left(\partial_{i} \tilde{c}\right)^{2} \underbrace{\sum_{j} \partial_{j}(u)_{j}}_{=0} \\
& \leq \frac{1}{4} \int_{\Omega}\left|D^{2} \tilde{c}\right|^{2}+\int_{\Omega} \tilde{c}^{2}|\nabla u|^{2} \leq \frac{1}{4} \int_{\Omega}\left|D^{2} \tilde{c}\right|^{2}+\tilde{C} \int_{\Omega}|\nabla u|^{2}
\end{aligned}
$$

for some $\tilde{C}>0$ in combination with Young's inequality and the boundedness of $\tilde{c}$. Applying Young's inequality once more and using the embedding $W^{2,2}(\Omega) \subset W^{1,4}(\Omega)$ in spatial dimension 2 or 3 concludes the proof.

By means of the last lemma, we have reduced the ill-signed term of the $n \log n$ estimate to some integrals containing the flow $u$. Fortunately, there is a well-known fact about the Navier-Stokes equation, which can be proved straightforwardly:

Lemma 3.3. There exist constants $C_{1}, C_{2}>0$ such that

$$
\frac{d}{d t} \int_{\Omega}|u|^{2}+2 v \int_{\Omega}|\nabla u|^{2}+C_{1} \int_{\Omega}|u|^{2} \leq C_{2} \int_{\Omega} n^{2} .
$$

Remark 3.4. The previous Lemma also covers the approximate Navier-Stokes equations for a finite $m$. In fact, it can be shown that the constants are independent of $m$.

Combining the last three lemmata, we can infer the following $n \log n$ estimate. This estimate plays a crucial role in the proof of the global existence of a solution in spatial dimension two as well as in spatial dimension three. For $N=3$, we will use an integrated version of this estimate in order to prove a uniform bound of an appropriate sequence of the desired solution. In contrast, we require a differential form in $N=2$ since the estimate contains an information of $\int_{\Omega}|\nabla c|^{4}$ which will be useful later on (see Lemma 4.1).

\section{Corollary 3.5. We introduce the continuous function}

$$
z(t):=\int_{\Omega} n^{2}(t)+\int_{\Omega} \frac{|\nabla n(t)|^{2}}{n(t)+e}+\int_{\Omega}\left|D^{2} c(t)\right|^{2}+\int_{\Omega}|\nabla c(t)|^{4}+\int_{\Omega}|\nabla u(t)|^{2}
$$


and define

$$
y(t):=\int_{\Omega}(n(t)+e)(\log (n(t)+e)-1)+C_{0} \int_{\Omega}|\nabla \tilde{c}(t)|^{2}+C_{1} \int_{\Omega}|u(t)|^{2}
$$

for some given parameters $C_{0}, C_{1}>0$. Then y is differentiable and bounded. In addition, there exist positive constants $C_{2}, C_{3}$ and an appropriate choice of $C_{0}, C_{1}$ such that y fulfils

$$
y^{\prime}+C_{2} z \leq-C_{2} y+C_{3}+\frac{1}{2} \int_{\Omega}(l(n)-r) \log (n+e) .
$$

Proof. According to the previous three lemmata, we find some positive constants $C_{2}, C_{3}$ such that

$$
\begin{array}{r}
y^{\prime}+C_{2}\left(\int_{\Omega} n^{2}+\int_{\Omega} \frac{|\nabla n|^{2}}{n+e}+\int_{\Omega}\left|D^{2} \tilde{c}\right|^{2}+\int_{\Omega}|\nabla \tilde{c}|^{4}+\int_{\Omega}|\nabla u|^{2}\right) \\
\leq-C_{2} y+\frac{1}{2} \int_{\Omega}(l(n)-r) \log (n+e)+C_{3}
\end{array}
$$

holds. Since $(l(n)-r)$ is negative or zero, we infer from Grönwall's inequality that $y$ is bounded. Now we apply the transformation $\tilde{c}:=e^{-g_{1}}\left(c-g_{2}\right)$ to the integrals containing $\nabla \tilde{c}$ and $D^{2} \tilde{c}$. Since $g_{1}, g_{2} \in C^{2}(\bar{\Omega} \times[0, \infty))$ are fixed, we obtain the inequality of the assertion after changing the constants.

\section{2. $L^{p}$ estimates}

So far, we have proved an $n \log n$ type Lyapunov estimate. The next step is to derive an estimate for $n^{p}$. A straightforward argument leads to

Lemma 3.6. Let $p>1$; then there exist positive constants $C_{0}, C_{1}$ such that we have

$$
\frac{1}{p} \frac{d}{d t} \int_{\Omega} n^{p}+\frac{3(p-1)}{4} \int_{\Omega}|\nabla n|^{2} n^{p-2} \leq C_{0} \int_{\Omega}|\nabla c|^{2 p+2}+C_{1}-\int_{\Omega} n^{p} .
$$

Proof. We multiply equation (cNS) $)_{1}$ by $n^{p-1}$ and integrate by parts to entail

$$
\frac{1}{p} \frac{d}{d t} \int_{\Omega} n^{p}=-(p-1) \int_{\Omega}|\nabla n|^{2} n^{p-2}+(p-1) \int_{\Omega} \chi(c) \nabla c \nabla n n^{p-1}+\int_{\Omega} l(n) n^{p-1} .
$$

In order to cope with the middle unsigned term, we use Young's inequality repeatedly and the fact that $c$ is bounded as well as that $l(n)$ admits the upper bound $r-d n^{2}(\mathrm{cf} .(8))$.

In spatial dimension two, we may use the Gagliardo-Nirenberg inequalities to ameliorate the previous proof.

Lemma 3.7. If $N=2$ and $p>1$, then there exists a constant $C_{p}>0$ such that

$$
\frac{1}{p} \frac{d}{d t} \int_{\Omega} n^{p}+\frac{p-1}{2} \int_{\Omega}|\nabla n|^{2} n^{p-2} \leq C_{p}\left(\int_{\Omega}|\nabla c|^{4}+1\right) \int_{\Omega} n^{p}+\int_{\Omega} l(n) n^{p-1}
$$

holds.

Remark 3.8. The assertion is restricted to spatial dimension two, because of a special use of the Gagliardo-Nirenberg inequalities, which depend on the dimension. This concept is introduced by Winkler in [32] in the proof of Lemma 4.5. 
Proof. In contrast to the proof of Lemma 3.6, we estimate

$$
\int_{\Omega} \chi(c) n^{p-1} \nabla c \cdot \nabla n \leq \frac{p-1}{2} \int_{\Omega}|\nabla n|^{2} n^{p-2}+\frac{C}{p-1}\left(1+\int_{\Omega}|\nabla c|^{4}\right) \int_{\Omega} n^{p}
$$

for some $C>0$ depending on $p$. In order to show this, we begin with using Young's and Cauchy-Schwarz' inequality to see

$$
(p-1) \int_{\Omega} \chi(c) \nabla c \nabla n n^{p-1} \leq \frac{(p-1)^{2}}{4} \int_{\Omega}|\nabla n|^{2} n^{p-2}+C_{0} \sqrt{\int_{\Omega}|\nabla c|^{4} \int_{\Omega} n^{2 p}}
$$

for some $C_{0}>0$ since $c$ is bounded. We set $z:=n^{\frac{p}{2}}$ and obtain a constant $C_{1}>0$ from Gagliardo-Nirenberg satisfying

$$
\begin{aligned}
\left(\int_{\Omega} n^{2 p}\right)^{\frac{1}{2}}=\|z\|_{L^{4}(\Omega)}^{2} & \leq C_{1}\left(\|\nabla z\|_{L^{2}(\Omega)}\|z\|_{L^{2}(\Omega)}+\|z\|_{L^{2}(\Omega)}^{2}\right) \\
& =C_{1}\left(\frac{p}{2}\left\|\nabla n n^{\frac{p}{2}-1}\right\|_{L^{2}(\Omega)}\|n\|_{L^{p}(\Omega)}^{\frac{p}{2}}+\|n\|_{L^{p}(\Omega)}^{p}\right) .
\end{aligned}
$$

Noting $\sqrt{x} \leq 1+x$ for $x>0$, we observe that

$$
\begin{aligned}
& \sqrt{\int_{\Omega}|\nabla c|^{4} \int_{\Omega} n^{2 p}} \leq \frac{1}{4}(p-1)^{2} \int_{\Omega}|\nabla n|^{2} n^{p-2}+\frac{C_{1}^{2} p^{2}}{4(p-1)^{2}} \int_{\Omega}|\nabla c|^{4} \int_{\Omega} n^{p} \\
& +C_{1}\left(1+\int_{\Omega}|\nabla c|^{4}\right)\|n\|_{L^{p}(\Omega)}^{p}
\end{aligned}
$$

holds thanks to Young's inequality. Finally, we set $C:=C_{0} C_{1}\left(1+\frac{C_{1} p^{2}}{4(p-1)^{2}}\right)$ to verify the estimation above, which implies the assertion using the same methods as in the proof of Lemma 3.6.

As we observe in the two former lemmata, an estimate on $\int_{\Omega} n^{p}$ includes an integral either of $|\nabla c|^{2 p+2}$ or of $|\nabla c|^{4}$. This motivates the following investigation:

Lemma 3.9. Let $p \geq 1$, then there exist some constants $C_{1}, \ldots, C_{4}>0$ depending on $p$ such that we have

$$
\frac{d}{d t} \int_{\Omega}|\nabla \tilde{c}|^{2 p}+C_{1} \int_{\Omega}\left|D^{2} \tilde{c}\right|^{2}|\nabla \tilde{c}|^{2 p-2}+C_{2} \int_{\Omega}|\nabla c|^{2 p+2} \leq C_{2} \int_{\Omega}|u|^{2 p+2}+C_{3} \int_{\Omega} n^{p+1}+C_{4} .
$$

Proof. This proof is based upon the proof of Lemma 2.2 from [31]. Recalling Lemma 2.5 and inequalities in (24), we have $\tilde{h}^{2} \leq C\left(1+|u|^{2}+n^{2}+|\nabla \tilde{c}|^{2}\right)$, since $\tilde{c}$ is bounded, $h, g_{1}, g_{2}, f$ are smooth and $\tilde{n}, \tilde{c}$ can be estimated using the definition of the backward transformation $T$. Now, we can rewrite the equation for $\tilde{c}$ as

$$
\tilde{c}_{t}=\Delta \tilde{c}-u \cdot \nabla \tilde{c}+\tilde{h}(\tilde{n}, \tilde{c}, \nabla \tilde{c}, u)=: \Delta \tilde{c}+\eta(\tilde{n}, \tilde{c}, \nabla \tilde{c}, u) .
$$

We multiply the equation for $\tilde{c}$ by $\Delta \tilde{c}|\nabla \tilde{c}|^{2 r}$ and arrive at

$$
\begin{aligned}
\frac{1}{2 r} \frac{d}{d t} \int_{\Omega}|\nabla \tilde{c}|^{2 r}=-\int_{\Omega}(\Delta \tilde{c}+\eta) \Delta \tilde{c}|\nabla \tilde{c}|^{2 r-2}-\int_{\Omega}(\Delta \tilde{c}+\eta) \nabla \tilde{c} \cdot \nabla|\nabla \tilde{c}|^{2 r-2} \\
=\int_{\Omega} \nabla \Delta \tilde{c} \cdot \nabla \tilde{c}|\nabla \tilde{c}|^{2 r-2}-\int_{\Omega}\left(\Delta \tilde{c}|\nabla \tilde{c}|^{2 r-2}+\nabla \tilde{c} \cdot \nabla|\nabla \tilde{c}|^{2 r-2}\right) \eta,
\end{aligned}
$$


where we have used integration by parts twice and the fact that $\frac{\partial \tilde{c}}{\partial v}=0$ on $\partial \Omega$. Continuing, we estimate the two integrals separately. For this, we apply the identity $\Delta|\nabla \tilde{c}|^{2}=2\left|D^{2} \tilde{c}\right|^{2}+2 \nabla \tilde{c} \cdot \nabla \Delta \tilde{c}$ to the first integral on the right-hand side. Recalling that $\frac{\partial \tilde{c}}{\partial \nu}=0$ holds on $\partial \Omega$, we use integration by parts to see

$$
\int_{\Omega} \nabla \Delta \tilde{c} \cdot \nabla \tilde{c}|\nabla \tilde{c}|^{2 r-2}+\int_{\Omega}\left|D^{2} \tilde{c}\right|^{2}|\nabla \tilde{c}|^{2 r-2}=-\left.\left.\frac{r-1}{2} \int_{\Omega}|\nabla| \nabla \tilde{c}\right|^{2}\right|^{2}|\nabla \tilde{c}|^{2 r-4}+I
$$

with $I:=\int_{\partial \Omega} \frac{\partial|\nabla \tilde{c}|^{2}}{\partial v}|\nabla \tilde{c}|^{2 r-2}$. According to [6], we deduce $\frac{\partial|\nabla \tilde{c}|^{2}}{\partial v} \leq 0$ and hence $I \leq 0$ from the facts that $\Omega$ is convex and $\frac{\partial \tilde{c}}{\partial \nu}=0$. Writing $\nabla \tilde{c} \cdot \nabla|\nabla \tilde{c}|^{2 r-2} \eta=(r-1) \nabla|\nabla \tilde{c}|^{2}|\nabla \tilde{c}|^{2 r-4} \cdot(\nabla \tilde{c} \eta)$, we estimate the integral concerning $\eta$ using Young's inequality and the relation $|\Delta \tilde{c}|^{2} \leq N\left|D^{2} \tilde{c}\right|^{2}$ to conclude

$$
\frac{1}{2 r} \frac{d}{d t} \int_{\Omega}|\nabla \tilde{c}|^{2 r}+\frac{4-N}{4} \int_{\Omega}\left|D^{2} \tilde{c}\right|^{2}|\nabla \tilde{c}|^{2 r-2} \leq \frac{r+1}{2} \int_{\Omega}|\nabla \tilde{c}|^{2 r-2}|\eta|^{2} .
$$

Splitting $\eta$ into $u \cdot \nabla \tilde{c}+\tilde{h}$ permits a more explicit estimate on $\nabla \tilde{c}$. For this purpose, we apply Young's inequality to deduce

$$
\begin{aligned}
& \frac{1}{2} \int_{\Omega}|\nabla \tilde{c}|^{2 r-2}|u \cdot \nabla \tilde{c}+\tilde{h}|^{2} \leq \int_{\Omega}|\nabla \tilde{c}|^{2 r-2}|u \cdot \nabla \tilde{c}|^{2}+\int_{\Omega}|\nabla \tilde{c}|^{2 r-2} \tilde{h}^{2} \\
& \leq \int_{\Omega}|\nabla \tilde{c}|^{2 r}|u|^{2}+\frac{r-1}{r+1} \int_{\Omega} \tilde{\varepsilon}|\nabla \tilde{c}|^{2 r+2}+\frac{2}{r+1} \int_{\Omega} \tilde{\varepsilon}^{\frac{1-r}{2}}|\tilde{h}|^{r+1} \\
& \quad \leq \frac{2 r-1}{r+1} \int_{\Omega} \tilde{\varepsilon}|\nabla \tilde{c}|^{2 r+2}+\frac{1}{r+1} \int_{\Omega} \tilde{\varepsilon}^{-r}|u|^{2 r+2}+\frac{2}{r+1} \int_{\Omega} \tilde{\varepsilon}^{\frac{1-r}{2}}|\tilde{h}|^{r+1}
\end{aligned}
$$

for every measurable $\tilde{\varepsilon}: \Omega \rightarrow(0, \infty)$. In particular, defining $b=b(\tilde{c}):=8(N+2 r)^{2} \sup |\tilde{c}|^{2}$ and $\frac{1}{\tilde{\varepsilon}}:=(2 r-1) b$, we arrive at

$$
\frac{r+1}{2} \int_{\Omega}|\nabla \tilde{c}|^{2 r-2}|\eta|^{2} \leq \frac{1}{b} \int_{\Omega} \tilde{\varepsilon}|\nabla \tilde{c}|^{2 r+2}+C_{r} \int_{\Omega}\left(b^{r}|u|^{2 r+2}+b^{\frac{r-1}{2}}|\tilde{h}|^{r+1}\right)
$$

chosen $C_{r}>0$ adequate. So far, we have achieved an separation between $\tilde{c}$ and $u, \tilde{h}$ by introducing an ill-signed term involving the $(2 r+2)$ th power of $|\nabla \tilde{c}|$. Thus, the next step is to digest this term utilising the left-hand side of inequality (38). Integration by parts and the fact that $\frac{\partial \tilde{c}}{\partial \nu}=0$ on $\partial \Omega$ yield

$$
\int_{\Omega}|\nabla \tilde{c}|^{2 r+2}=-\int_{\Omega} \tilde{c} \Delta \tilde{c}|\nabla \tilde{c}|^{2 r}-r \int_{\Omega} \tilde{c} \nabla \tilde{c} \cdot \nabla|\nabla \tilde{c}|^{2}|\nabla \tilde{c}|^{2 r-2} .
$$

The identity $\nabla|\nabla z|^{2}=2 D^{2} z \nabla z$ and a second application of $|\Delta \tilde{c}|^{2} \leq N\left|D^{2} \tilde{c}\right|^{2}$ ensure in conjunction with CauchySchwarz's inequality

$$
\int_{\Omega}|\nabla \tilde{c}|^{2 r+2} \leq(N+2 r)\|\tilde{c}\|_{L^{\infty}(\Omega)}\left(\int_{\Omega}|\nabla \tilde{c}|^{2 r+2}\right)^{\frac{1}{2}}\left(\int_{\Omega}\left|D^{2} \tilde{c}\right|^{2}|\nabla \tilde{c}|^{2 r-2}\right)^{\frac{1}{2}} .
$$

Dividing by $\left(\int_{\Omega}|\nabla \tilde{c}|^{2 r+2}\right)^{\frac{1}{2}}$ and collecting the previous results end up at

$$
\frac{d}{d t} \int_{\Omega}|\nabla \tilde{c}|^{2 p}+C_{1} \int_{\Omega}\left|D^{2} \tilde{c}\right|^{2}|\nabla \tilde{c}|^{2 p-2}+C_{2} \int_{\Omega}|\nabla \tilde{c}|^{2 p+2} \leq C_{2} \int_{\Omega}|u|^{2 p+2}+C_{3} \int_{\Omega} n^{p+1}+C_{4}
$$

for some constants $C_{1}, \ldots, C_{4}>0$. We note that the third term on the left of the assertion contains the nontransformed $\nabla c$. Since $c, \tilde{c}$ are bounded and $g_{1}, g_{2}$ are smooth, we can use the formula $\nabla c e^{-g_{1}}=\nabla \tilde{c}+\tilde{c} \nabla g_{1}+$ $\nabla g_{2} e^{-g_{1}}$ to replace $|\nabla \tilde{c}|^{2 p+2}$ by the non-transformed $|\nabla c|^{2 p+2}$ in the estimate after adjusting the constants $C_{2}$ and $C_{4}$. This is possible, because $g_{1}$ and $g_{2}$ have been fixed at the beginning of Section 2 . 


\subsection{Estimates for $\nabla n$}

The investigation of the $L^{p}$ norm of $n$ in the last subsection results in a high dependency on $p$ of the constants. Therefore, we cannot take the limit in $p$ to gain an upper bound of $n$. Gagliardo-Nirenberg's inequality, however, allows us to estimate the $L^{\infty}$ norm of $n$ if we are able to control its $W^{1, p}$ norm for $p>N$. Since the equations for $c$ and $n$ are somehow similar, we would like to estimate the gradient of $n$ likewise to Lemma 3.9. In contrast to $c$ and $\tilde{c}$, neither the $L^{\infty}$ norm of $n$ nor of $\tilde{n}$ is finite a priori, which leads to the following lemma.

Lemma 3.10. For every $\varepsilon>0$ and $p \geq 1$, there exist some positive constants $C_{0}, C_{1}, C_{2}$ and $P$ fulfilling

$$
\begin{aligned}
\frac{1}{2 p} \frac{d}{d t} \int_{\Omega}|\nabla \tilde{n}|^{2 p}+C_{0} \int_{\Omega}\left|D^{2} \tilde{n}\right|^{2}|\nabla \tilde{n}|^{2 p-2}+C_{1} \int_{\Omega} \frac{|\nabla \tilde{n}|^{2 p+2}}{(\tilde{n}+1)^{2}} \\
\quad \leq C_{2} \int_{\Omega}|u|^{2 p+2+\varepsilon}+C_{2} \int_{\Omega}|\nabla \tilde{c}|^{2 p+2+\varepsilon}+C_{2} \int_{\Omega}|\Delta \tilde{c}|^{p+1+\varepsilon}+C_{2} \int_{\Omega} \tilde{n}^{P}+C_{2} .
\end{aligned}
$$

Proof. First of all, we rewrite the third term on the left-hand side by computing

$$
\int_{\Omega} \frac{\nabla \tilde{n} \cdot \nabla \tilde{n}}{(\tilde{n}+1)^{2}}|\nabla \tilde{n}|^{2 p}=\int_{\Omega} \frac{1}{\tilde{n}+1} \Delta \tilde{n}|\nabla \tilde{n}|^{2 p}+p \int_{\Omega} \frac{1}{\tilde{n}+1} \nabla \tilde{n} \cdot \nabla|\nabla \tilde{n}|^{2}|\nabla \tilde{n}|^{2 p-2},
$$

where we have used integration by parts and $\frac{\partial \tilde{n}}{\partial v}=0$ on $\partial \Omega$. As in the proof of Lemma 3.9, we use $\nabla|\nabla \tilde{n}|^{2}=2 D^{2} \tilde{n} \nabla \tilde{n}$ and $|\Delta \tilde{n}| \leq N\left|D^{2} \tilde{n}\right|$ to infer

$$
\int_{\Omega} \frac{|\nabla \tilde{n}|^{2 p+2}}{(\tilde{n}+1)^{2}} \leq(N+2 p) \int_{\Omega} \frac{1}{\tilde{n}+1}\left|D^{2} \tilde{n}\right||\nabla \tilde{n}|^{2 p} .
$$

By means of the Cauchy-Schwarz inequality, this implies

$$
\int_{\Omega} \frac{|\nabla \tilde{n}|^{2 p+2}}{(\tilde{n}+1)^{2}} \leq(N+2 p)^{2} \int_{\Omega}\left|D^{2} \tilde{n}\right|^{2}|\nabla \tilde{n}|^{2 p-2} .
$$

Putting $f_{1}:=u+\tilde{\mathbf{a}}(\tilde{c}, \nabla \tilde{c})$ and $f_{2}:=\tilde{n} \nabla \cdot(\tilde{\mathbf{a}}(\tilde{c}, \nabla \tilde{c}))+\tilde{R}(\tilde{n}, \tilde{c}, \tilde{\mathbf{a}}(\tilde{c}, \nabla \tilde{c}))+\tilde{l}(\tilde{n}, \tilde{c})$, we obtain

$$
\begin{aligned}
\frac{1}{2 p} \frac{d}{d t} \int_{\Omega} & |\nabla \tilde{n}|^{2 p}+\frac{1}{8} \int_{\Omega}\left|D^{2} \tilde{n}\right|^{2}|\nabla \tilde{n}|^{2 p-2} \\
& \leq C_{1} \int_{\Omega}(\tilde{n}+1)^{2 p}\left|f_{1}\right|^{2 p+2}+C_{2} \int_{\Omega}(\tilde{n}+1)^{p-1}\left|f_{2}\right|^{p+1}
\end{aligned}
$$

for some $C_{0}, C_{1}, C_{2}>0$ similarly to the proof of Lemma 3.9 (cf. Inequalities (38) and (39) with $(2 p-1) \tilde{\varepsilon}=\frac{1}{8}(N+$ $2 p)^{-2}(\tilde{n}+1)^{-2}$ and $\left.1=(2 r-1) \tilde{\varepsilon} b\right)$. Note that the constants $C_{0}, C_{1}, C_{2}$ may depend on $p$.

In order to estimate the right-hand side, we recall $\tilde{\mathbf{a}}$ and $\tilde{l}$ :

$$
\begin{aligned}
\tilde{\mathbf{a}}(x, t, \tilde{c}, \nabla \tilde{c}) & =-\chi\left(T_{c}(\tilde{c})\right)\left(\nabla \tilde{c}+\tilde{c} \nabla g_{1}+\nabla g_{2} e^{-g_{1}}\right) e^{g_{1}}, \\
\tilde{l}(\tilde{n}, \tilde{c}) & =A\left(T_{c}(\tilde{c})\right) l\left(T_{n}(\tilde{n}, \tilde{c})\right),
\end{aligned}
$$

where $T_{c}$ and $T_{n}$ are defined in Definition 2.3. By the boundedness of $\tilde{c}$ and the smoothness of $g_{1}, g_{2}$, we have

$$
|\tilde{\mathbf{a}}(\tilde{c}, \nabla \tilde{c})| \leq C_{3}(|\nabla \tilde{c}|+1) \quad \text { and } \quad|\nabla \cdot \tilde{\mathbf{a}}(\tilde{c}, \nabla \tilde{c})| \leq C_{3}\left(|\nabla \tilde{c}|^{2}+|\Delta \tilde{c}|+1\right)
$$

The conditions of the parameter functions (8)-(12) on page 1017 permit only a polynomial growth for $l$ implying $\tilde{l}(\tilde{c}, \tilde{n}) \leq C_{4}\left(1+|\tilde{n}|^{Q}\right)$ (here $Q>0$ is the same as in $\left.(10)\right)$, since $\tilde{c}$ is bounded. Moreover, we have

$$
|\tilde{R}(\tilde{n}, \tilde{c}, \tilde{\mathbf{a}}(\tilde{c}, \nabla \tilde{c}))| \leq C_{5}\left(\tilde{n}+|\nabla \tilde{c}|^{2}+1\right) \tilde{n}
$$

in accord with Inequalities (23). The prove can be completed using Young's inequality. 


\section{Global solvability of the chemotaxis-Navier-Stokes equations}

The local existence result - Proposition 2.6 - admits a blow-up criterion to determine whether the local solution can or cannot be extended globally. Given the parameter functions as in (8)-(12), we assume that the chemotaxis-NavierStokes equations (cNS) are classically solvable on $\bar{\Omega} \times\left[0, T_{\max }\right)$ for some $T_{\max } \in(0, \infty]$ with solution $(n, c, u, P)$. Here, we choose $T_{\max }$ maximal with respect to the blow-up criterion of Proposition 2.6, which implies

$$
\|n(t)\|_{L^{\infty}(\Omega)}+\|\nabla n(t)\|_{L^{2}(\Omega)}+\|c(t)\|_{W^{1,4}(\Omega)}+\left\|A^{\alpha} u(t)\right\|_{L^{2}(\Omega)} \rightarrow \infty \text {, as } t \nearrow T_{\max }
$$

unless $T_{\max }=\infty$. Throughout this section, we attempt to bound each summand in (42), which will be done separately for the cases $N=2$ and $N=3$ in Section 4.1 and 4.2, respectively. Unfortunately, in spatial dimension three, we are only able to prove a global existence for an approximated system. However, an appropriate energy type estimate permits passing the limit to a weak solution of the Problem (cNS).

\subsection{The two-dimensional case}

In this section, we show that the term (42) remains bounded on $t \in(0, T]$ for every finite $T \leq T_{\max }$ in the case $N=2$. Note that this results in a global in-time existence since it is only permitted for $T_{\max }=\infty$.

During the following calculation, we fix a finite $T \leq T_{\max }$ and bound the term (42) stepwise. We estimate the $L^{\infty}$ norm of $n$ using Gagliardo-Nirenberg's inequality

$$
\|n\|_{L^{\infty}(\Omega)} \leq C\left(\|n\|_{L^{p}(\Omega)}+\|\nabla n\|_{L^{p}(\Omega)}\right)
$$

for $p=N+1$ and some $C>0$. In addition, the summand $\left\|A^{\alpha} u(t)\right\|_{L^{2}(\Omega)}$ can be bounded in terms of $\|n\|_{L^{2}(\Omega)}$ (cf. [32] Eq. (4.19), pages 339-341). This allows us to neglect the term concerning $u$ in (42). Moreover, the fact that $\alpha>\frac{1}{2}$ by assumption entails that $u$ is locally bounded (see [32,25]).

Since $c$ is bounded according to Lemma 1.7, we introduce the new blow-up criterion for finite $T_{\max }$ which reads

$$
\|n(t)\|_{L^{p}(\Omega)}+\|\nabla n(t)\|_{L^{p}(\Omega)}+\|\nabla c(t)\|_{L^{4}(\Omega)} \rightarrow \infty \text { as } t \nearrow T_{\max }
$$

for some $p=N+1$.

This new criterion guides us to continue in three steps, i.e. estimating each term separately. As we observe later on, the most appropriate way is to begin with the $L^{p}$ norm of $n$ and to prove an estimate for the gradient of $c$ before concentrating on $\nabla n$.

Lemma 4.1. $\int_{\Omega} n^{p}$ is bounded on $\left[0, T_{\max }\right)$.

Proof. Let $C_{p}$ denote the constant from Lemma 3.7. By means of Lemma 3.7, we estimate the time derivative of $\int_{\Omega} n^{p}$ by

$$
\frac{d}{d t} \int_{\Omega} n^{p} \leq p C_{p} \int_{\Omega}|\nabla c|^{4} \int_{\Omega} n^{p}-(1+\beta) \int_{\Omega} n^{p}+\gamma
$$

for some $\beta, \gamma>0$ (depending on $p$ ), where we have exploit $l(n) \leq r-d n^{2}$ and Young's inequality. If we directly apply Grönwall's inequality, we obtain an exponential bound. Therefore, we need a better approach to show global boundedness: Let us recall $y$ from Corollary 3.5, which is bounded fulfilling the differential inequality

$$
y^{\prime} \leq-C \int_{\Omega}|\nabla c|^{4}-\frac{1}{\alpha} y+\alpha
$$

for some $C, \alpha>0$. Defining $z:=\frac{p}{C} C_{p} y$, we analyse by means of Eq. (44) that

$$
\frac{d}{d t}\left(e^{z} \int_{\Omega} n^{p}\right)=e^{z} \frac{d}{d t} \int_{\Omega} n^{p}+z^{\prime} e^{z} \int_{\Omega} n^{p} \leq\left(-\int_{\Omega} n^{p}+\gamma\right) e^{z}=-e^{z} \int_{\Omega} n^{p}+\delta
$$

holds for some constant $\delta>\gamma e^{z}$. This constant exists since $y$ and hence $z$ are globally bounded. Due to Grönwall's inequality, $e^{z} \int_{\Omega} n^{p}$ is bounded as well as $\int_{\Omega} n^{p}$, because of the non-negativity of $z$. 
The $L^{p}$ regularity of $n$ we have just won admits the following lemma concerning the gradient of $c$ :

Lemma 4.2. $\int_{\Omega}|\nabla c(t)|^{p}$ is bounded on $[0, T]$ if $2 \leq p \leq 10$ and $T<T_{\max }$.

Proof. First, we recall a short version of Lemma 3.9: There exist some constants $C_{1}, \ldots, C_{3}>0$ depending on $p \geq 2$ such that

$$
\frac{1}{p} \frac{d}{d t} \int_{\Omega}|\nabla \tilde{c}|^{p} \leq C_{1} \int_{\Omega}|u|^{p+1}+C_{2} \int_{\Omega} n^{\frac{p}{2}+1}+C_{3}
$$

holds. The fact that $\int_{\Omega} n^{q}$ is bounded on $\left[0, T_{\max }\right]$ for all $q \geq 1$ can be used twice: On the one hand, this clearly implies the boundedness of the integral containing $n$ by setting $q=\frac{p}{2}+1$. On the other hand, $u$ is bounded on $\bar{\Omega} \times(0, T)$ according to the boundedness of $\|n\|_{L^{2} \Omega}$ as seen above. Thus, $\int_{\Omega}|\nabla \tilde{c}|^{p}$ is locally bounded in $t$ whenever $\int_{\Omega}\left|\nabla \tilde{c}_{0}\right|^{p}<\infty$. Finally the assertion is shown using $C_{4}|\nabla c| \leq|\nabla \tilde{c}|+C_{5}$ and $C_{6}|\nabla \tilde{c}| \leq|\nabla c|+C_{7}$ for some $C_{4}, \ldots, C_{7}>0$.

Before being able to estimate the gradient of $n$, we need to ascertain more about $\Delta \tilde{c}$ and $\nabla \tilde{n}$ in some $L^{q}$ space. We cannot expect $\|\Delta \tilde{c}(t)\|_{L^{p}}$ and $\|\nabla n(t)\|_{L^{p}}$ to be bounded near $t=0$ since the initial values are not sufficiently regular. Therefore, let $0<T_{0}<T<T_{\max }$.

Lemma 4.3. $\Delta \tilde{c}$ belongs to $L^{q}\left(\bar{\Omega} \times\left(T_{0}, T\right)\right)$ for all $1<q \leq 10$.

Proof. We rewrite the equation for $\tilde{c}$ as $\tilde{c}_{t}=\Delta \tilde{c}+f$, for some functions $f_{1}, f_{2}$. Making use of Lemmata 4.1 and 4.2 as well as the fact that $u$ is bounded on $\Omega \times(0, T)$, we can show that $f \in L^{q}(\Omega \times(0, T))$ holds for $q \leq 10$. The maximal Sobolev regularity (cf. [16] Theorem 1 and Corollary 1.1) applied to the heat semigroup with Neumann boundary conditions guarantees that $\Delta \tilde{c}$ also belongs to $L^{q}\left(\Omega \times\left(T_{0}, T\right)\right)$ for all $0<T_{0}<T$ as required.

Lemma 4.4. $\int_{\Omega}|\nabla \tilde{n}|^{p}$ is bounded on $\left(T_{0}, T\right)$ for every $2 \leq p<8$.

Proof. Likewise to the estimates of $\nabla c$, we work in the transformed system $\tilde{n}$ and formulate a version of Corollary 3.10: Let $\varepsilon$ be positive; then there exist constants $C_{1}, P>0$ depending on $p$ and $\varepsilon$ such that we have

$$
\begin{aligned}
\frac{1}{p} \frac{d}{d t} \int_{\Omega}|\nabla \tilde{n}|^{p} \leq C_{1} \int_{\Omega}|u|^{p+2+\varepsilon}+C_{1} \int_{\Omega}|\nabla \tilde{c}|^{p+2+\varepsilon} & \\
& +C_{1} \int_{\Omega}|\Delta \tilde{c}|^{\frac{p}{2}+1+\varepsilon}+C_{1} \int_{\Omega} \tilde{n}^{P}+C_{1} \int_{\Omega} \tilde{n}^{\frac{p}{2}-1} .
\end{aligned}
$$

We choose $\varepsilon>0$ such that $p+2+\varepsilon \leq 10$. We proceed similarly to the proof of Lemma 4.2: Since $\int_{\Omega}|\nabla \tilde{c}|^{10}$, $\int_{T_{0}}^{t} \int_{\Omega}|\Delta \tilde{c}|^{10}$ and $\int_{\Omega} n^{q}$ are bounded, the term $\int_{\Omega}|\nabla \tilde{n}(t)|^{p}$ is bounded as well if $\int_{\Omega}\left|\nabla \tilde{n}\left(T_{0}\right)\right|^{p}<\infty$.

Although the relation $\tilde{n}=n A(c)$ between $n$ and $\tilde{n}$ contains a dependency on $c$, we can estimate $\nabla n$ in terms of $n$, $c, \nabla c, \nabla \tilde{n}$.

\subsection{Global weak solution in spatial dimension three}

The global existence in three dimensions requires a more delicate proof and only results in a weak solvability. The main reason for these difficulties is the dimension dependency of the Gagliardo-Nirenberg inequalities. Therefore, we reconsider two concepts of the proof which succeed in spatial dimension two:

1. The proof of a global bound for the solution of the Navier-Stokes equations is still an open problem in the three-dimensional case. This is the main reason why we can only expect a weak solvability. A standard way to achieve this is to approximate the Navier-Stokes system with a Galerkin method of restricting the problem to the eigenspaces of the Stokes operator (see [5] Theorem (Leray)). 
2. Unfortunately, the proof of the global boundedness of $\int_{\Omega} n^{p}(t)$ in spatial dimension two fails in the threedimensional case. Since our aim is already reduced to find a weak solution, we approximate the equation concerning $n$ in order to fix this issue and replace $l$ by

$$
l_{\varepsilon}(x):=l(x)-\varepsilon x^{3} \quad \text { for all } x>0
$$

and $\varepsilon \in(0,1)$. We underline the necessity of a uniform bound while dealing with approximate equations. Subsection 3.1 providing an $n \log n$ argument has discovered an energy estimate so far which is independent of the exact formula of $l_{\varepsilon}$ and holds uniformly for all $\varepsilon \in(0,1)$.

Our approximate system is finally given by

$$
\left\{\begin{array}{rlrl}
n_{t}+u \cdot \nabla n & =\Delta n-\nabla \cdot(n \chi(c) \nabla c)+l_{\varepsilon}(n), & x \in \Omega, t>0, \\
c_{t}+u \cdot \nabla c & =\Delta c-n f(c)+h(x, t, c), & x \in \Omega, t>0, & x \in \\
u_{t} & =-A u+P^{m} B(u \cdot \nabla u)+P^{m} \mathcal{P} n \nabla \varphi, & & t>0, \\
(n, c)(x, 0) & =\left(n_{0}, c_{0}\right)(x), & x \in \Omega, \\
u(0) & =P^{m} u_{0} \in L_{\sigma}^{2} & & x \in \partial \Omega, t>0, \\
\frac{\partial c}{\partial v}(x, t) & =\frac{\partial g_{1}}{\partial v}(x)\left(c(x, t)-g_{2}(x, t)\right), & & x \in \partial \Omega, t>0
\end{array}\right.
$$

for $\varepsilon \in(0,1)$ and $m \in \mathbb{N}$, where $n_{0}, c_{0}, u_{0}$ are as in (13).

\subsubsection{Global solvability in $3 D$ of an approximate system}

The approximate system defined above possesses a local solution on a maximal interval $\left[0, T_{\max }\right)$ with its blow-up criterion

$$
\|n(t)\|_{L^{p}(\Omega)}+\|\nabla n(t)\|_{L^{p}(\Omega)}+\|\nabla c(t)\|_{L^{4}(\Omega)} \rightarrow \infty \text { as } t \nearrow T_{\max }
$$

for $p=N+1$ unless $T_{\max }=\infty$, which can be derived similarly to the two-dimensional case. We have already seen that the transformed system has the same blow-up criterion as the standard system. We only have to guarantee that $n$ is bounded in $L^{\infty}$ and that $\nabla n$ possesses the same $L^{p}$ regularity as $\nabla c$ in both systems. Thus, we will prove the $L^{p}$ boundedness of $n$ in the non-transformed system and the rest in the transformed system as a matter of convenience.

Lemma 4.5. Given $p>1$ and finite $0<T \leq T_{\max }$, there exists a constant $C=C(\varepsilon, m)$ such that we have

$$
\int_{\Omega} n^{p}(x, t) d x<C \text { and } \int_{\Omega}|\nabla \tilde{c}|^{2 p}(x, t) d x<C .
$$

Proof. The proof is similar as in the two dimensional case, where we have to use Lemma 3.6 instead of Lemma 3.7. Note that in this case the constant $C$ depends on $T_{\max }$.

The boundedness of $\nabla \tilde{n}$ in $L^{\infty}\left(0, T ; L^{4}(\Omega)\right)$ is deduced exactly as in the two-dimensional case (see Lemma 4.4). We only have to observe, that $u_{m}$ belongs to the linear span of the first $m$ eigenfunctions of Stokes operator $A=-\mathcal{P} \Delta$. Therefore, $\left\|A^{k} u\right\|_{L_{\sigma}^{2}(\Omega)} \leq C\left\|A^{k} u_{m}\right\|_{L_{\sigma}^{2}(\Omega)}$ for some $C_{m}>0$. Since $\partial_{t}\left\|u_{m}\right\|_{L_{\sigma}^{2}(\Omega)}$ can be bounded by $c_{1}\|n\|_{L^{2}(\Omega)}+c_{2}$ for some $c_{1}, c_{2}>0$, the global existence result of the local boundedness of $\int_{0}^{t} \int_{\Omega} n^{2}$ is bounded with the aid of the $n \log n$ energy estimate (see Corollary 3.5).

Proposition 4.6. The system (46) admits a global classical solution. 


\subsubsection{Uniform bounds for the approximating sequence}

Corollary 4.7. Fix any $t>0$ and let $n_{\varepsilon, m}, c_{\varepsilon, m}, u_{\varepsilon, m}$ be a solution of $(46)$ on $[0, \infty)$, where $\varepsilon \in(0,1)$ and $m \in \mathbb{N}$. Thus,

1. $\left(n_{\varepsilon, m}\right)_{\varepsilon, m}$ is a bounded sequence in $L^{2}(\Omega \times) \cap L^{\frac{4}{3}}\left(0, t ; W^{1, \frac{4}{3}}(\Omega)\right)$. Furthermore,

$$
\int_{0}^{t} \int_{\Omega} \frac{\left|\nabla n_{\varepsilon, m}\right|^{2}}{n_{\varepsilon, m}+e}, \int_{\Omega} n_{\varepsilon, m}(t) \log \left(n_{\varepsilon, m}(t)+e\right), \int_{0}^{t} \int_{\Omega}\left(r-l_{\varepsilon}\left(n_{\varepsilon, m}\right)\right) \log \left(n_{\varepsilon, m}+e\right)
$$

are uniformly bounded in $(\varepsilon, m)$.

2. $\left(c_{\varepsilon, m}\right)_{\varepsilon, m}$ is bounded in $L^{4}\left(0, t ; W^{1,4}(\Omega)\right) \cap L^{2}\left(0, t ; W^{2,2}(\Omega)\right)$ and in $L^{\infty}(\Omega \times(0, \infty))$.

3. $\left(u_{\varepsilon, m}\right)_{\varepsilon, m}$ is bounded in $L^{\infty}\left(0, \infty ; L_{\sigma}^{2}(\Omega)\right) \cap L^{2}\left(0, t ; W^{1,2}(\Omega)\right) \cap L^{\frac{10}{3}}(\Omega \times(0, t))$.

Proof. This corollary is an almost direct consequence of Corollary 3.5 in combination with Remark 3.4 and Grönwall's inequality. The only missing ingredient is to show the boundedness of

$$
\int_{0}^{t} \int_{\Omega}|\nabla n|^{\frac{4}{3}} \text { and } \int_{0}^{t} \int_{\Omega}|u|^{\frac{10}{3}}
$$

whereas we write $n=n_{\varepsilon, m}$ and $u=u_{\varepsilon, m}$ here and below. First, we directly deduce that

$$
\begin{aligned}
\iint_{0}^{t}|\nabla n|^{\frac{4}{3}} & =\int_{|\nabla n|^{\frac{4}{3}}>(n+e)^{2}}|\nabla n|^{\frac{4}{3}}+\int_{|\nabla n|^{\frac{2}{3}} \leq(n+e)}|\nabla n|^{\frac{4}{3}} \\
& =\int_{0}^{t} \int_{\Omega} \frac{|\nabla n|^{2}}{(n+e)}+\iint_{0}^{t}(n+e)^{2}
\end{aligned}
$$

is bounded. According to Gagliardo-Nirenberg, it holds

$$
\int_{0}^{t} \int_{\Omega}|u|^{\frac{10}{3}}=\int_{0}^{t}\|u\|_{L^{\frac{10}{3}}(\Omega)}^{\frac{10}{3}} \leq \int_{0}^{t}\|u\|_{W^{1,2}(\Omega)}^{\frac{10}{3} \alpha}\|u\|_{L^{2}(\Omega)}^{\frac{10}{3}(1-\alpha)}
$$

for $\alpha=\frac{3}{2}-\frac{9}{10}=\frac{3}{5}$. Finally, the boundedness of $\int_{0}^{t} \int_{\Omega}|\nabla u|^{\frac{10}{3} \alpha}=\int_{0}^{t} \int_{\Omega}|\nabla u|^{2}$ and $\sup _{s} \int_{\Omega}|u|^{2}(s)$ imply that $\int_{0}^{t} \int_{\Omega}|u|^{\frac{10}{3}}$ is also bounded.

Lemma 4.8. Fix $t>0$ and let $n_{\varepsilon, m}, c_{\varepsilon, m}, u_{\varepsilon, m}$ be a solution of $(46)$ on $[0, \infty)$, where $\varepsilon \in(0,1)$ and $m \in \mathbb{N}$. Then

1. $\left(\partial_{t} n_{\varepsilon, m}\right)$ is uniformly bounded in $L^{1}\left(0, t ;\left(W^{1,4}(\Omega)\right)^{\prime}\right)$.

2. $\left(\partial_{t} c_{\varepsilon, m}\right)$ is uniformly bounded in $L^{\frac{4}{3}}(\Omega \times(0, t))$.

3. $\left(\partial_{t} u_{\varepsilon, m}\right)$ is uniformly bounded in $L^{\frac{4}{3}}\left(0, t ;\left(W_{\sigma}^{1,2}(\Omega)\right)^{\prime}\right)$.

Proof. Throughout this proof, we omit again the indices $\varepsilon, m$.

1. We multiply equation $(46)_{1}$ by $\xi \in W^{1,4}(\Omega)$ and integrate by parts to obtain

$$
\int_{\Omega} n_{t} \xi=\int_{\Omega} u n \cdot \nabla \xi+\int_{\Omega} \nabla n \cdot \nabla \xi+\int_{\Omega} n \nabla c \cdot \nabla \xi+\int_{\Omega} l_{\varepsilon}(n) \xi .
$$


In spatial dimension three, $L^{\infty}(\Omega)$ is embedded in $W^{1,4}(\Omega)$. Therefore, in conjunction with Hölder's inequality and Young's inequality, we have

$$
\left\|n_{t}\right\|_{W^{1,4}(\Omega)^{\prime}}=\sup _{\xi \in W^{1,4}(\Omega)} \frac{\left|\int_{\Omega} n_{t} \xi\right|}{\|\xi\|_{W^{1,4}(\Omega)}} \leq\|n\|_{L^{2}}^{2}+\|u\|_{L^{4}}^{2}+\|\nabla c\|_{L^{4}}^{2}+\|\nabla n\|_{L^{\frac{4}{3}}}^{\frac{4}{3}}+\|\nabla c\|_{L^{4}}^{4}+C\left\|l_{\varepsilon}(n)\right\|_{L^{1}}
$$

for some $C>0$ writing $L^{p}=L^{p}(\Omega)$. The $L^{4}$-norm of $u$ can be estimated by using the Gagliardo-Nirenberg inequality, namely

$$
\|u\|_{L^{4}(\Omega)} \leq\|u\|_{W^{1,2}(\Omega)}^{\frac{3}{4}}\|u\|_{L^{2}(\Omega)}^{\frac{1}{4}} .
$$

Lastly, we integrate the norm of $n_{t}$ in time to see

$$
\begin{aligned}
\left\|n_{t}\right\|_{L^{1}\left(0, t ; W^{1,4}(\Omega)^{\prime}\right)} \leq \int_{0} \int_{\Omega} n^{2}+\sup _{0 \leq s \leq t} \int_{\Omega}|u(s)|^{2} & \int_{0}^{t}\|u\|_{W^{1,2}(\Omega)}^{\frac{3}{2}} \\
& +\int_{0}^{t}\|\nabla n\|_{L^{\frac{4}{3}}(\Omega)}^{\frac{4}{3}}+2 \int_{0}^{t}\|\nabla c\|_{L^{4}(\Omega)}^{4}+\int_{0}^{t}\left\|l_{\varepsilon}(n)\right\|_{L^{1}(\Omega)} .
\end{aligned}
$$

Due to Corollary 4.7, the right-hand side is bounded, which entails the first assertion of Lemma 4.8.

2. Recall the equation for $\tilde{c}$, which reads

$$
\tilde{c}_{t}=-u \cdot \nabla \tilde{c}+\Delta \tilde{c}+2 \nabla g_{1} \cdot \nabla \tilde{c}+\tilde{h}(\tilde{n}, \tilde{c}, u):=\Delta \tilde{c}+F_{\varepsilon, m},
$$

whereas we may estimate $\left|F_{\varepsilon, m}\right| \leq C\left(1+|u|^{\frac{3}{2}}+n+|\nabla \tilde{c}|^{3}\right)$ for some $C>0$. Due to Corollary $4.7, F_{\varepsilon, m}$ is bounded in $L^{\frac{4}{3}}(\Omega \times(0, t))$ such that we deduce the existence of a $\tilde{C}_{t}>0$ fulfilling $\left\|\tilde{c}_{t}\right\|_{L^{\frac{4}{3}}(\Omega \times(0, t))} \leq \tilde{C}_{t}$ from the maximal Sobolev regularity ([16] Theorem 1 and Corollary 1.1 applied to the heat semi-group).

3. This claim is a direct consequence of [5] Lemma 8.1 in conjunction with the boundedness of $n$ in $L^{2}(\Omega \times(0, t))$ according to Corollary 4.7 .

\subsubsection{Precompactness of the approximating sequence}

Fix $t>0$ and let $n_{\varepsilon, m}, c_{\varepsilon, m}, u_{\varepsilon, m}$ be a classical solution of $(46)$ on $[0, \infty)$, where $\varepsilon \in(0,1)$ and $m \in \mathbb{N}$.

\section{Lemma 4.9. The sequence}

1. $\left(n_{\varepsilon, m}\right)$ is precompact in $L^{\frac{4}{3}}(\Omega \times(0, t))$ as well as

2. $\left(c_{\varepsilon, m}\right)$ is precompact in $L^{2}\left(0, t ; W^{1,2}(\Omega)\right)$ and in $C^{0}\left([0, t] ; L^{2}(\Omega)\right)$.

3. Finally, $\left(u_{\varepsilon, m}\right)$ is precompact in $L^{2}\left(0, t ; L_{\sigma}^{2}(\Omega)\right)$.

Proof. This lemma is a direct consequence of the Aubin-Lions Lemma (see [24] Theorem 5 and Corollary 4), the properties of $n_{\varepsilon, m}, c_{\varepsilon, m}, u_{\varepsilon, m}$ stated in Corollary 4.7 and Lemma 4.8 as well as the Rellich-Kondrachov theorem ([11] Section 5.7, Theorem 1 and the following remark).

Lemma 4.10. The sequence $\left(h\left(c_{\varepsilon_{k}, m_{k}}\right)\right)$ is precompact in $L^{1}(\Omega \times(0, \infty))$ and bounded in $L^{p}(\Omega \times(0, \infty))$ for $p<\infty$.

Proof. The precompactness of $\left(h\left(c_{\varepsilon_{k}, m_{k}}\right)\right)$ in $L^{1}$ follows from the fact, that $h\left(c_{\varepsilon_{k}, m_{k}}\right)$ is bounded in $L^{\infty}$ and Lebesgue's convergence theorem. This and the uniform boundedness of $\left(h\left(c_{\varepsilon_{k}, m_{k}}\right)\right)$ yield the boundedness of $\left(h\left(c_{\varepsilon_{k}, m_{k}}\right)\right)$ in $L^{p}$ for $p<\infty$.

Lemma 4.11. The sequence $l_{\varepsilon}\left(n_{\varepsilon, m}\right)$ is weakly precompact in $L^{1}(\Omega \times(0, t))$. 
Proof. First, Corollary 4.7 yields that $l_{\varepsilon}\left(n_{\varepsilon, m}\right)$ is bounded in $L^{1}(\Omega \times(0, t))$. By Dunford-Pettis (see [29] Definition VIII.6.6 and Proposition VIII.6.9), it remains to show that $l_{\varepsilon}\left(n_{\varepsilon, m}\right)$ is uniform integrable, which we postpone to Lemma 4.14.

Lemma 4.12 (de la Vallée-Poussin). Let $\Omega \subset \mathbb{R}^{N}$ be bounded and $\Theta:[0, \infty) \rightarrow[0, \infty]$ be an increasing function with $\frac{\Theta(t)}{t} \rightarrow \infty$ for $t \rightarrow \infty$. A bounded subset $\mathcal{F} \subset L^{1}(\Omega)$ is uniform integrable if there exists a constant $C>0$ such that

$$
\int_{\Omega} \Theta(|f|)<C
$$

for all $f \in \mathcal{F}$.

Proof. See [18] Theorem T22.

Before proving the uniform integrability of $l_{\varepsilon}\left(n_{\varepsilon}\right)$, we consider an auxiliary function in the following lemma which we will require during the proof.

Lemma 4.13. Let $\psi:[0, \infty) \rightarrow[0, \infty)$ be locally bounded and fulfil $\psi(t) \rightarrow \infty$ as $t \rightarrow \infty$. We define an almost inverse function of $\psi$ by

$$
\hat{\psi}(y):=\inf \psi^{-1}([y, \infty)) \quad \text { for } y \geq 0 .
$$

Clearly, if $\psi$ is bijective, then $\hat{\psi}=\psi^{-1}$. In general, $\hat{\psi}:[0, \infty) \rightarrow[0, \infty)$ is well-defined, non-decreasing and satisfies

$$
\lim _{t \rightarrow \infty} \hat{\psi}(t) \rightarrow \infty \quad \text { as well as } \quad \hat{\psi} \circ \psi(t) \leq t \text { for } t \geq 0 \text {. }
$$

Proof. A direct calculation provides the well-definedness of $\psi$ and equation (51). The remaining property $\psi(t) \rightarrow \infty$ as $t \rightarrow \infty$ can be proved by contradiction and is left to the reader.

Lemma 4.14. The sequence $\left(l_{\varepsilon}\left(n_{\varepsilon, m}\right)\right)$ is uniform integrable on $\Omega \times(0, t)$ for $0<\varepsilon \leq 1$ and $m \in \mathbb{N}$.

Proof. Our plan is to use Lemma 4.12 to obtain that $\left(l_{\varepsilon}\left(n_{\varepsilon, m}\right)\right)$ is uniform integrability. Therefore, we have to find a function $\Theta$ as in Lemma 4.12. This will be done separately for the two terms of $l_{\varepsilon}\left(n_{\varepsilon, m}\right)$. Recall that we have

$$
l_{\varepsilon}\left(n_{\varepsilon, m}\right)=l\left(n_{\varepsilon, m}\right)-\varepsilon n_{\varepsilon, m}^{3} .
$$

To begin with, let us consider the first term $l\left(n_{\varepsilon, m}\right)$ and define $\psi(t):=r-l(t) \geq d t^{2}$. From the conditions in (8), we infer that $\psi$ is non-negative and $\psi(t) \rightarrow \infty$ as $t \rightarrow \infty$. Therefore, we set $\hat{\psi}$ as in Lemma 4.13 and obtain the stated properties of $\hat{\psi}$. These preliminary considerations let us introduce the desired function

$$
\Theta(t):=t \log (\hat{\psi}(t)+e) .
$$

In order to continue, we have to check the two conditions for $\Theta$ from Lemma 4.12. Since $\hat{\psi}(t) \rightarrow \infty$ as $t \rightarrow \infty$, we infer that $\Theta(t) / t=\log (\hat{\psi}(t)+e) \rightarrow \infty$ as $t \rightarrow \infty$. Furthermore, in view of $\psi(t):=r-l(t) \geq 0$, we deduce

$$
\begin{aligned}
& \int_{\Omega} \Theta\left(\psi\left(n_{\varepsilon, m}\right)\right)=\int_{\Omega} \psi\left(n_{\varepsilon, m}\right) \log \left(\hat{\psi}\left(\psi\left(n_{\varepsilon, m}\right)\right)+e\right) \\
& \quad \leq \int_{\Omega} \psi\left(n_{\varepsilon, m}\right) \log \left(n_{\varepsilon, m}+e\right)=\int_{\Omega}\left(r-l\left(n_{\varepsilon, m}\right)\right) \log \left(n_{\varepsilon, m}+e\right) \leq C .
\end{aligned}
$$

Thus, Lemma 4.12 yields that $\psi\left(n_{\varepsilon, m}\right)=r-l\left(n_{\varepsilon, m}\right)$ is uniform integrable. It remains to show the uniform integrability of $\varepsilon n_{\varepsilon, m}^{3}$. We define $\tilde{\Theta}(t):=t \log (\sqrt[3]{t}+e)$ and see 


$$
\begin{aligned}
\tilde{\Theta}\left(\varepsilon n_{\varepsilon, m}^{3}\right) & =\varepsilon n_{\varepsilon, m}^{3} \log \left(\sqrt[3]{\varepsilon} n_{\varepsilon, m}+e\right) \\
& \leq \varepsilon n_{\varepsilon, m}^{3} \log \left(n_{\varepsilon, m}+e\right) \leq\left(r-l_{\varepsilon}\left(n_{\varepsilon, m}\right)\right) \log \left(n_{\varepsilon, m}+e\right),
\end{aligned}
$$

for $\varepsilon \leq 1$. Similarly as before, we can verify the two conditions for $\tilde{\Theta}$ from Lemma 4.12 such that we obtain the uniform integrability of $\varepsilon n_{\varepsilon, m}^{3}$ from Lemma 4.12 .

\subsubsection{Proof of Theorem 1.3}

Let $n_{\varepsilon, m}, c_{\varepsilon, m}, u_{\varepsilon, m}$ be a classical solution of $(46)$ on $[0, \infty)$ where $\varepsilon \in(0,1)$ and $m \in \mathbb{N}$.

Lemma 4.15. Let $(n, c, u)$ be almost everywhere a pointwise limit point of $\left(n_{\varepsilon, m}, c_{\varepsilon, m}, u_{\varepsilon, m}\right)$. Then there exists a sequence $\left(\varepsilon_{k}, m_{k}\right) \rightarrow(0, \infty)$ such that

$$
\begin{aligned}
u_{\varepsilon_{k}, m_{k}} n_{\varepsilon_{k}, m_{k}} & \rightarrow \text { un } \quad \text { in } L^{\frac{5}{4}}(\Omega \times(0, t)), \\
n_{\varepsilon_{k}, m_{k}} \chi\left(c_{\varepsilon_{k}, m_{k}}\right) \nabla c_{\varepsilon_{k}, m_{k}} & \rightarrow n \chi(c) \nabla c \quad \text { in } L^{\frac{4}{3}}(\Omega \times(0, t)), \\
n_{\varepsilon_{k}, m_{k}} f\left(c_{\varepsilon_{k}, m_{k}}\right) & \rightarrow n f(c) \quad \text { in } L^{2}(\Omega \times(0, t)), \\
u_{\varepsilon_{k}, m_{k}} \cdot \nabla c_{\varepsilon_{k}, m_{k}} & \rightarrow u \cdot \nabla c \quad \text { in } L^{\frac{20}{11}}(\Omega \times(0, t))
\end{aligned}
$$

for all $t>0$.

Proof. The sequence $\left(n_{\varepsilon_{k}, m_{k}} u_{\varepsilon_{k}, m_{k}}\right)$ is bounded in $L^{\frac{5}{4}}(\Omega \times(0, t))$ and therefore weakly precompact, since Young's inequality implies

$$
\int_{\Omega}\left|n_{\varepsilon_{k}, m_{k}} u_{\varepsilon_{k}, m_{k}}\right|^{\frac{5}{4}} \leq \frac{5}{8} \int_{\Omega} n_{\varepsilon_{k}, m_{k}}^{2}+\frac{3}{8} \int_{\Omega}\left|u_{\varepsilon_{k}, m_{k}}\right|^{\frac{10}{3}},
$$

which is bounded due to Corollary 4.7. Hence, a subsequence of $\left(u_{\varepsilon_{k}, m_{k}} n_{\varepsilon_{k}, m_{k}}\right)$ converges weakly to un. The remaining convergences are proved similarly by extracting a subsequence. Here, we use that

$$
\int_{\Omega}\left|n_{\varepsilon_{k}, m_{k}} \chi\left(c_{\varepsilon_{k}, m_{k}}\right) \nabla c_{\varepsilon_{k}, m_{k}}\right|^{\frac{4}{3}} \leq \frac{2}{3} \int_{\Omega}\left|n_{\varepsilon_{k}, m_{k}}\right|^{2}+\frac{1}{3}\left\|\chi\left(c_{\varepsilon_{k}, m_{k}}\right)\right\|_{L^{\infty}(\Omega)}^{4} \int_{\Omega}\left|\nabla c_{\varepsilon_{k}, m_{k}}\right|^{4}
$$

is bounded (see Corollary 4.7) as well as

$$
\begin{aligned}
& \int_{\Omega}\left|n_{\varepsilon_{k}, m_{k}} f\left(c_{\varepsilon_{k}, m_{k}}\right)\right|^{2} \leq\left\|f\left(c_{\varepsilon_{k}, m_{k}}\right)\right\|_{L^{\infty}(\Omega)}^{2} \int_{\Omega}\left|n_{\varepsilon_{k}, m_{k}}\right|^{2}, \\
& \int_{\Omega}\left|u_{\varepsilon_{k}, m_{k}} \cdot \nabla c_{\varepsilon_{k}, m_{k}}\right|^{\frac{20}{11}} \leq \frac{6}{11} \int_{\Omega}\left|u_{\varepsilon_{k}, m_{k}}\right|^{\frac{10}{3}}+\frac{5}{11} \int_{\Omega}\left|\nabla c_{\varepsilon_{k}, m_{k}}\right|^{4} .
\end{aligned}
$$

Proposition 4.16. There exist

$$
\begin{aligned}
& n \in L_{l o c}^{2}\left(0, \infty ; L^{2}(\Omega)\right) \cap L_{l o c}^{\frac{4}{3}}\left(0, \infty ; W^{1, \frac{4}{3}}(\Omega)\right), \\
& c \in L^{\infty}(\Omega \times(0, \infty)) \cap L_{l o c}^{2}\left(0, \infty ; W^{2,2}(\Omega)\right) \cap W_{l o c}^{1, \frac{4}{3}}(\Omega \times[0, \infty)) \cap C^{0}\left([0, \infty) ; L^{2}(\Omega)\right), \\
& u \in L^{\infty}\left(0, \infty ; L_{\sigma}^{2}(\Omega)\right) \cap L_{l o c}^{2}\left(0, \infty ; W_{\sigma}^{1,2}(\Omega)\right)
\end{aligned}
$$

such that

$$
\begin{aligned}
\int_{0}^{t} \int_{\Omega} n \Psi_{t}+\int_{0}^{t} \int_{\Omega} n u \cdot \nabla \Psi & +\int_{0}^{t} \int_{\Omega} \nabla n \cdot \nabla \Psi \\
& =\int_{\Omega} n_{0} \Psi(0, \cdot)-\int_{0}^{t} \int_{\Omega} n \chi(c) \nabla c \cdot \nabla \Psi+\int_{0}^{t} \int_{\Omega} l(n) \Psi
\end{aligned}
$$




$$
\int_{0}^{t} \int_{\Omega} u \xi_{t}+\int_{0}^{t} \int_{\Omega} u \otimes u: \nabla \xi+v \int_{0}^{t} \int_{\Omega} \nabla u: \nabla \xi=\int_{\Omega} u_{0} \cdot \xi(0, \cdot)+\iint_{0}^{t} n \nabla \varphi \cdot \xi
$$

for all $\Psi \in C_{c}^{\infty}(\bar{\Omega} \times[0, \infty))$ and $\xi \in C_{c}^{\infty}(\bar{\Omega} \times[0, \infty))^{N}$ with $\nabla \cdot \xi=0$.

Proof. Testing equations (46) $)_{1}$ and (46) 3 by $\Psi$ and $\xi$, respectively, and using integration by parts, this proposition is consequence of Corollary 4.7 and Lemmata $4.9,4.11$ and 4.15 .

Likewise to the proof of the previous proposition, we can show the following proposition making additionally use of Lemma 4.9.

Proposition 4.17. The function $(n, c, u)$ in Proposition 4.16 can be chosen such that we have

$$
c_{t}+u \cdot \nabla c=\Delta c-n f(c)+h(c)
$$

in $L_{l o c}^{\frac{4}{3}}\left(0, \infty ; L^{\frac{4}{3}}(\Omega)\right)$ and $c(0, \cdot)=c_{0}$ in $L^{2}(\Omega)$. In addition, $c$ fulfils the boundary condition

$$
\frac{\partial c}{\partial v}=\frac{\partial g_{1}}{\partial v}\left(c-g_{2}\right)
$$

in $L_{l o c}^{2}\left(0, \infty ; L^{2}(\partial \Omega)\right)$.

Remark 4.18. Since $c$ is a strong solution of (55), it is also a weak one as asserted in Theorem 1.3.

Finally, Theorem 1.3 is a consequence of Propositions 4.16 and 4.17.

\section{Conflict of interest statement}

We confirm that there are no known conflicts of interest associated with this publication and there has been no significant financial support for this work that could have influenced its outcome.

\section{Acknowledgement}

This work has emerged from the author's M.Sc. thesis under the supervision of M. Winkler. The author is grateful for helpful advice and constant support.

\section{References}

[1] Masashi Aida, Atsushi Yagi, Global attractor for approximate system of chemotaxis and growth, Dyn. Contin. Discrete Impuls. Syst., Ser. A, Math. Anal. (ISSN 1201-3390) 10 (1-3) (2003) 309-315, 1918-2538/e.

[2] Peter William Atkins, Julio de Paula, Physical Chemistry, 8th edition, Oxford University Press, 2006.

[3] Vincent Calvez, José A. Carrillo, Volume effects in the Keller-Segel model: energy estimates preventing blow-up, J. Math. Pures Appl. (9) (ISSN 0021-7824) 86 (2) (2006) 155-175, http://dx.doi.org/10.1016/j.matpur.2006.04.002.

[4] Myeongju Chae, Kyungkeun Kang, Jihoon Lee, Global existence and temporal decay in Keller-Segel models coupled to fluid equations, Commun. Partial Differ. Equ. (ISSN 0360-5302) 39 (7) (2014) 1205-1235, http://dx.doi.org/10.1080/03605302.2013.852224, 1532-4133/e.

[5] Peter Constantin, Ciprian Foias, Navier-Stokes Equations, University of Chicago Press, Chicago, IL etc., ISBN 0-226-11549-6, 1988.

[6] Roberta Dal Passo, Harald Garcke, Günther Grün, On a fourth-order degenerate parabolic equation: global entropy estimates, existence, and qualitative behavior of solutions, SIAM J. Math. Anal. (ISSN 0036-1410) 29 (2) (1998) 321-342, http://dx.doi.org/10.1137/ S0036141096306170, 1095-7154/e.

[7] Marco Di Francesco, Alexander Lorz, Peter Markowich, Chemotaxis-fluid coupled model for swimming bacteria with nonlinear diffusion: global existence and asymptotic behavior, Discrete Contin. Dyn. Syst. (ISSN 1078-0947) 28 (4) (2010) 1437-1453, http://dx.doi.org/ 10.3934/dcds.2010.28.1437, 1553-5231/e.

[8] Renjun Duan, Zhaoyin Xiang, A note on global existence for the chemotaxis-Stokes model with nonlinear diffusion, Int. Math. Res. Not. (ISSN 1073-7928) 2014 (7) (2014) 1833-1852, http://dx.doi.org/10.1093/imrn/rns270, 1687-0247/e.

[9] Renjun Duan, Alexander Lorz, Peter Markowich, Global solutions to the coupled chemotaxis-fluid equations, Commun. Partial Differ. Equ. (ISSN 0360-5302) 35 (9) (2010) 1635-1673, http://dx.doi.org/10.1080/03605302.2010.497199, 1532-4133/e. 
[10] Elio Espejo, Takashi Suzuki, Reaction terms avoiding aggregation in slow fluids, Nonlinear Anal., Real World Appl. (ISSN 1468-1218) 21 (2015) 110-126, http://dx.doi.org/10.1016/j.nonrwa.2014.07.001.

[11] Lawrence C. Evans, Partial Differential Equations, 2nd edition, American Mathematical Society, Providence, RI, ISBN 978-0-8218-4974-3, 2010.

[12] Avner Friedman, Partial Differential Equations, Holt, Rinehart and Winston, Inc., New York etc., 1969, 262 pp.

[13] H. Fujita, Tosio Kato, On the Navier-Stokes initial value problem. I, Arch. Ration. Mech. Anal. (ISSN 0003-9527) 16 (1964) 269-315, http://dx.doi.org/10.1007/BF00276188, 1432-0673/e.

[14] Dan Henry, Geometric Theory of Semilinear Parabolic Equations, Lecture Notes in Mathematics, vol. 840, Springer-Verlag. IV, BerlinHeidelberg-New York, 1981.

[15] O.A. Ladyzhenskaya, V.A. Solonnikov, N.N. Ural'tseva, Linear and Quasi-Linear Equations of Parabolic Type, translated from the Russian by S. Smith, Translations of Mathematical Monographs, vol. 23, American Mathematical Society (AMS). XI, Providence, RI, 1968,648 pp.

[16] Damien Lamberton, Equations d'évolution linéaires associées à des semi-groupes de contractions dans les espaces $L^{p}$ (Evolution equations associated to contraction semigroups in $L^{p}$ spaces), J. Funct. Anal. (ISSN 0022-1236) 72 (1987) 252-262, http://dx.doi.org/10.1016/ 0022-1236(87)90088-7.

[17] J.L. Lions, E. Magenes, Problèmes aux limites non homogenes et applications, vol. 1, 1968.

[18] Paul-André Meyer, Probability and Potentials, Blaisdell Publishing Co., 1966.

[19] Masayasu Mimura, Tohru Tsujikawa, Aggregating pattern dynamics in a chemotaxis model including growth, Phys. A, Stat. Mech. Appl. (ISSN 0378-4371) 230 (3-4) (1996) 499-543, http://dx.doi.org/10.1016/0378-4371(96)00051-9.

[20] Xavier Mora, Semilinear parabolic problems define semiflows on $C^{k}$ spaces, Trans. Am. Math. Soc. (ISSN 0002-9947) 278 (1983) 21-55, http://dx.doi.org/10.2307/1999300, 1088-6850/e.

[21] Toshitaka Nagai, Takasi Senba, Kiyoshi Yoshida, Application of the Trudinger-Moser inequality to a parabolic system of chemotaxis, Funkc. Ekvacioj, Ser. Int. (ISSN 0532-8721) 40 (3) (1997) 411-433.

[22] Koichi Osaki, Atsushi Yagi, Global existence for a chemotaxis-growth system in $\mathbb{R}^{2}$, Adv. Math. Sci. Appl. (ISSN 1343-4373) 12 (2) (2002) 587-606.

[23] Koichi Osaki, Tohru Tsujikawa, Atsushi Yagi, Masayasu Mimura, Exponential attractor for a chemotaxis-growth system of equations, Nonlinear Anal., Theory Methods Appl. (ISSN 0362-546X) 51 (1) (2002) 119-144, http://dx.doi.org/10.1016/S0362-546X(01)00815-X.

[24] Jacques Simon, Compact sets in the space $L^{p}(0, T ; B)$, Ann. Mat. Pura Appl. (4) (ISSN 0373-3114) 146 (1987) 65-96, http://dx.doi.org/ 10.1007/BF01762360, 1618-1891/e.

[25] Hermann Sohr, The Navier-Stokes Equations. An Elementary Functional Analytic Approach, Birkhäuser, Basel, ISBN 3-7643-6545-5, 2001.

[26] Youshan Tao, Michael Winkler, Global existence and boundedness in a Keller-Segel-Stokes model with arbitrary porous medium diffusion, Discrete Contin. Dyn. Syst. (ISSN 1078-0947) 32 (5) (2012) 1901-1914, http://dx.doi.org/10.3934/dcds.2012.32.1901, 1553-5231/e.

[27] Youshan Tao, Michael Winkler, Locally bounded global solutions in a three-dimensional chemotaxis-Stokes system with nonlinear diffusion, Ann. Inst. Henri Poincaré, Anal. Non Linéaire (ISSN 0294-1449) 30 (1) (2013) 157-178, http://dx.doi.org/10.1016/j.anihpc.2012.07.002.

[28] Idan Tuval, Luis Cisneros, Christopher Dombrowski, Charles W. Wolgemuth, John O. Kessler, Raymond E. Goldstein, Bacterial swimming and oxygen transport near contact lines, Proc. Natl. Acad. Sci. USA (ISSN 0027-8424) 102 (7) (2005) 2277-2282, http://dx.doi.org/ 10.1073/pnas.0406724102, 1091-6490/e.

[29] Werner Dirk, Funktionalanalysis, 7th revised and expanded edition, Springer, Berlin, ISBN 978-3-642-21016-7, 2011, 978-3-642-21017-4/ ebook.

[30] Michael Winkler, Aggregation vs. global diffusive behavior in the higher-dimensional Keller-Segel model, J. Differ. Equ. (ISSN 00220396) 248 (12) (2010) 2889-2905, http://dx.doi.org/10.1016/j.jde.2010.02.008.

[31] Michael Winkler, Boundedness in the higher-dimensional parabolic-parabolic chemotaxis system with logistic source, Commun. Partial Differ. Equ. (ISSN 0360-5302) 35 (8) (2010) 1516-1537, http://dx.doi.org/10.1080/03605300903473426, 1532-4133/e

[32] Michael Winkler, Global large-data solutions in a chemotaxis-(Navier-)Stokes system modeling cellular swimming in fluid drops, Commun. Partial Differ. Equ. (ISSN 0360-5302) 37 (1-3) (2012) 319-351, http://dx.doi.org/10.1080/03605302.2011.591865, 1532-4133/e.

[33] Michael Winkler, Global weak solutions in a three-dimensional chemotaxis-Navier-Stokes system, Ann. Inst. Henri Poincaré, Anal. Non Linéaire 33 (5) (2016) 1329-1352, http://dx.doi.org/10.1016/j.anihpc.2015.05.002.

[34] Michael Winkler, Boundedness and large time behavior in a three-dimensional chemotaxis-Stokes system with nonlinear diffusion and general sensitivity, Calc. Var. Partial Differ. Equ. (ISSN 0944-2669) 54 (4) (2015) 3789-3828, http://dx.doi.org/10.1007/s00526-015-0922-2, 1432-0835/e.

[35] Michael Winkler, A two-dimensional chemotaxis-Stokes system with rotational flux: global solvability, eventual smoothness and stabilization, preprint.

[36] Michael Winkler, Youshan Tao, Blow-up prevention by quadratic degradation in a two-dimensional Keller-Segel-Navier-Stokes system, preprint. 